\title{
Review Article \\ The Use of Nanoscaled Fibers or Tubes to Improve Biocompatibility and Bioactivity of Biomedical Materials
}

\author{
Xiaoming Li, ${ }^{1}$ Rongrong Cui, ${ }^{1}$ Wei Liu, ${ }^{1}$ Lianwen Sun, ${ }^{1}$ Bo Yu, ${ }^{2}$ Yubo Fan, \\ Qingling Feng, ${ }^{3}$ Fuzhai Cui, ${ }^{3}$ and Fumio Watari ${ }^{4}$ \\ ${ }^{1}$ Key Laboratory for Biomechanics and Mechanobiology of Ministry of Education, School of Biological Science and Medical Engineering, \\ Beihang University, Beijing 100191, China \\ ${ }^{2}$ Department of Orthopedics, Zhujiang Hospital of Southern Medical University, Guangzhou 510282, China \\ ${ }^{3}$ State Key Laboratory of New Ceramic and Fine Processing, Tsinghua University, Beijing 100084, China \\ ${ }^{4}$ Department of Biomedical Materials and Engineering, Graduate School of Dental Medicine, Hokkaido University, \\ Sapporo 060-8586, Japan
}

Correspondence should be addressed to Xiaoming Li; x.m.li@hotmail.com and Yubo Fan; yubofan@buaa.edu.cn

Received 13 July 2013; Accepted 22 August 2013

Academic Editor: Shuming Zhang

Copyright (C) 2013 Xiaoming Li et al. This is an open access article distributed under the Creative Commons Attribution License, which permits unrestricted use, distribution, and reproduction in any medium, provided the original work is properly cited.

Nanofibers and nanotubes have recently gained substantial interest for potential applications in tissue engineering due to their large ratio of surface area to volume and unique microstructure. It has been well proved that the mechanical property of matrix could be largely enhanced by the addition of nanoscaled fibers or tubes. At present, more and more researches have shown that the biocompatibility and bioactivity of biomedical materials could be improved by the addition of nanofibers or nanotubes. In this review, the efforts using nanofibers and nanotubes to improve biocompatibility and bioactivity of biomedical materials, including polymeric nanofibers/nanotubes, metallic nanofibers/nanotubes, and inorganic nanofibers/nanotubes, as well as their researches related, are demonstrated in sequence. Furthermore, the possible mechanism of improving biocompatibility and bioactivity of biomedical materials by nanofibers or nanotubes has been speculated to be that the specific protein absorption on the nanoscaled fibers or tubes plays important roles.

\section{Introduction}

In 2008, Williams redefined biocompatibility as follows [1]: biocompatibility refers to the ability of a biomaterial to perform its desired function with respect to a medical therapy, without eliciting any undesirable local or systemic effects in the recipient or beneficiary of that therapy but generating the most appropriate beneficial cellular or tissue response in that specific situation and optimising the clinically relevant performance of that therapy.

According to the ESB consensus conference of 1987 [2], a bioactive material is "one which has been designed to induce specific biological activity." Kokubo and Takadama consider bioactive materials as bone bonding materials in the beginning [3]; however, obviously, there has been a drift of meaning over time. The definition of both bioactive materials and bioactivity has been expanded. Bioactivity is considered as a property of having or producing an effect on living cell, tissue, and organ rather than only interacting with bone, which mainly displays in inducing specific protein or mineral absorption and cell differentiation, enhancing tissue growth and so on. In addition, bioactive materials have not only meant the bone bonding materials but also materials applied in tissue engineering application, biomedicine, drug delivery, and so on.

In recent years, more and more attention has been paid to the study of bioactive materials. Among all of the bioactive materials, the nanostructure materials occupy significant position. Nanomaterial is short for nanostructure material, which includes a natural, incidental, or manufactured material containing particles, in an unbound state or as an aggregate or as an agglomerate and where, for $50 \%$ or more of the particles in the number size distribution, one or more external dimensions is in the size range $1 \mathrm{~nm}-100 \mathrm{~nm}$. 
Nanomaterials have special mechanical, electrical, magnetic, optical, chemical, and other biological properties because of their high aspect ratio and surface area. Many nanomaterial surfaces exemplify high (bio and cyto) compatibility such as carbon nanotubes (CNTs), carbon nanofibers (CNFs), boron nitride nanotube (BNNTs), and other nanostructured materials, by promoting protein adsorption and enhancing subsequent cellular adhesion and tissue growth more than on conventional implant surfaces such as titanium, ceramics, and biopolymers. For instance, the bioactive-glass nanofibers have the ability to chemically bond with living bone tissue and have played a central role in the bone regeneration field, due to its excellent bioactivity, osteoconductivity, and even osteoinductivity [4], having been used in a variety of medical applications, such as implants in clinical bone repair and regeneration materials, bioactive coating of metallic implants in tissue engineering. Compared to graphite (GP), the multiwalled carbon nanotubes with higher aspect ratio and surface area have the ability to induce ectopic bone formation in the dorsal musculature of ddY mice. Moreover, multiwalled carbon nanotubes have the ability to induce osteogenic differentiation of human adipose-derived MSCs in vitro and ectopic bone formation in vivo [5]. The nanofibers and nanotubes occupy a significant position among lots of nanostructured materials due to the biocompatibility and bioactivity. Nanofibers and nanotubes are usually used for hard/soft-tissue application, such as bone or teeth repair and neuron injury repair. As it is known know that, many of these nanofibers or nanotubes have to interact with live cell, tissue, or organ directly, for one thing, the interaction between nanostructure biomaterials and cell, tissue, or organ must be safe; for another thing, it will be better if the interaction is positive.

Looking for novel nanofibers and nanotubes that have bioactive effect on cell, tissue, or organ and understanding the mechanism of the bioactive effect are meaningful. It will help to expand the application of nanomaterials that we have applied and enhance the nanomaterials' bioactive property. In this paper, first of all, nanofibers and nanotubes which have bioactive effect, including polymeric nanotubes and nanofibers, metallic nanotubes and nanofibers, and inorganic nanotubes and nanofibers, as well as the research related, are demonstrated in sequence.

\section{Bioactivity of Nanofibers}

\subsection{Inorganic Nanofibers}

2.1.1. Carbon Nanofibers. Throughout the various types of nanomaterials, carbon-based nanomaterials have been proved to be ideal for improving the microenvironment around proteins, since they are bioactive and biocompatible. CNFs, as one of the nanoscale carbonaceous materials, have recently attracted considerable attention owing to its unique mechanical, electrical, thermal, optical, and structural properties [6-9]. Compared with the CNTs which include singlewalled carbon nanotubes (SWNTs) and the multiwalled carbon nanotubes (MWNTs), CNFs have a larger diameter
(60-150 nm), a longer length $(30-100 \mu \mathrm{m})$, and a different surface morphology [10]. It is because of nanoeffect of carbon nanofibers, especially for very large surface area and unique microstructure that have extended the field of potential applications of CNFs as biomaterials.

However, poor dispersibility and chemical inertness of carbon nanofibers have largely restricted its applications especially when incorporating carbon nanofibers into composites. Surface functionalization is critical for resolving these problems, as surface-bound functional groups can enhance the wettability, dispersibility, and surface reactivity of carbon nanofibers and the degree of carbon/matrix interfacial binding within composite materials to some extent.

Two methods commonly employed to modify carbon nanostructure surfaces are covalent functionalization of surface carbon atom sites [11] and noncovalent wrapping of carbon nanostructures with surfactants, polymers, or ceramic coatings. CNFs, particularly those having the platelet or herringbone structures [12], possess edge-site carbon atoms of each graphene layer terminating the nanofiber surface and are more especially suitable for surface functionalization. Reactions between linker and matrix functional groups can afford carbon/matrix interfaces of high covalent integrity.

Carbon nanofibers, as one of the nanoscale carbonaceous materials, have attracted considerable attention due to the fact that they have more edge sites on the outer wall than carbon nanotubes, which can facilitate the electron transfer of electroactive analytes, such as proteins or enzymes, respectively $[13,14]$. Electrospinning has been demonstrated to be an efficient approach to produce continuous fibers with diameter ranging from tens of nanometers to several micrometers [15]. Electrospinning uses an electrical charge to draw very fine (typically on the micro- or nanoscale) fibres from a liquid. When a sufficiently high voltage is applied to a liquid droplet, the body of the liquid becomes charged, and electrostatic repulsion counteracts the surface tension and the droplet is stretched; at a critical point a stream of liquid erupts from the surface. Electrospinning method can not only be used for the fabrication of fibers but also for tubes. Generally speaking, CNFs produced by electrospinning method have several remarkable characteristics such as a very large ratio of surface area to volume and flexibility for functionalization, which make them an attractive biomaterial in biotechnology and medicine [14-18]. Carbon nanofibers can be also produced by vapor growth method or plasma enhanced chemical vapor depositing method and some other synthesis methods [19].

As mentioned above, the CNFs functionalization process aims at enhancing the solubility of CNFs and conjugating CNFs with bioactive molecules. In the following section we will briefly discuss some recent biomedical applications of CNFs. Usually CNFs can be used in bone regeneration and neural applications. One of the first studies using CNFs in scaffolds for tissue regeneration was the study of Price et al. [20]. For applications such as scaffolds for bone regeneration, CNFs can be ideal candidates due to their excellent mechanical properties and bioactivity. Rajzer et al. [21] produced carbon nanofibers from modified electrospun PAN/hydroxyapatite precursors and generated 
carbon nanofibers/HAp bioactive nanofibrous scaffold for bone tissue engineering. Rajzer et al. demonstrated this new material can lead to the development of a nanofibrous implants which can establish direct chemical bonds with bone tissue after implantation. Moreover, Bencano [22] used carbon fiber implants to fill osteochondral defects created on the articular surface of the patella of rabbits and studied the long-term histological changes of the repair process. They demonstrated that carbon fibers can be used to repair articular cartilage defects in the patella. Similarly, Elisa et al. [23] also illustrated carbon nanofibers clearly represented a unique and promising class of orthopedic/dental implant formulations with improved osseointegrative properties.

As an emerging interdisciplinary field, neural tissue engineering has evoked increasing interest from scientists wishing to develop novel and improved biological scaffolds that restore, maintain, or improve neural tissue functions. Since natural neural tissues have numerous nanostructured features, CNFs which also have such nanofeatures and exceptional electrical, mechanical, and biocompatible properties are excellent candidates for neural tissue repair. CNFs have aroused much interest in regenerative neural tissue engineering applications. It is reported that the astrocyte adhesion and proliferation were decreased on CNFs and $\mathrm{CNF} /$ polycarbonate urethane composites and consequently reduced the formation of glial scar tissue $[24,25]$. Vertically aligned CNFs coated with thin conductive polymers are developed to improve mechanical and electrical properties of CNFs and contribute to forming an intimate neuralelectrical interface between cells and nanofibers. Carbon fibers are particularly attractive for use in neural biomaterials not only due to these special properties but also due to their high conductivity and bioactivity. The functionalized Carbon fibers exhibit great potential implications in tissue engineering.

2.1.2. Phosphate Glass Nanofibers. Over the past decades, bioactive glass has played a central role in the bone regeneration field, due to its excellent bioactivity, osteoconductivity, and even osteoinductivity [4]. Because such materials have the ability to chemically bond with living bone tissue, they have been used in a variety of medical applications, such as implants in clinical bone repair and regeneration materials, bioactive coating of metallic implants in tissue engineering, tumor treatment, and protein and/or cell activation [26]. Most studies in this field have focused on meltderived glasses, either in the bulk or granular form. When formulated with biodegradable polymers, the potential of these bioactive-glass nanofibers to act as a tissue-engineering scaffold should be further increased by adopting the shape flexibility of polymers while retaining optimized mechanical properties (toughness, strength, and elastic modulus) and without sacrificing its excellent bioactivity. However, with the melt-spinning approach, the fiber diameter is limited to such micrometer scale (ca. tens to hundreds of micrometers) because of the associated processing restrictions [27]. Compared to melt-spinning approach, electrospinning is regarded as a simple and versatile method to produce ultrafine fibers with diameters ranging from microns down to a few nanometers; a variety of materials can be electrospun to form uniform fibers, such as organic, inorganic, and hybrid polymers (organic-inorganic composites) [28, 29]. Thus, nanofibrous scaffolds with various diameters can be obtained by employing an electrospinning process based on a sol-gel precursor [24].

For hard-tissue applications, such as the regeneration and repair of bones and teeth, several bioactive or bioinert materials have been used clinically. Kouhi et al. [30] prepared poly ( $\varepsilon$-caprolactone) (PCL)/bioactive glass (BG) composites nanofibers using electrospinning techniques, and they demonstrated this novel composites nanofibrous web was shown to have the ability to form a hydroxyapatite layer in the biological fluid on the nanofibers surface, which indicates their good potential for bone regeneration applications. Most of vivo studies on these bioglasses have confirmed their excellent biocompatibility with hard and even soft tissues. This is attributed mainly to their ability to form a bioactive layer at the interface in contact with living tissues, namely, the hydroxycarbonate apatite (HCA) layer, which is equivalent to the mineral phase of human hard tissues. Based on extensive research conducted in vitro and in vivo, bioactive glasses are considered as one of the most promising biomaterials for the "next generation" [4].

\subsection{Bioactivity of Polymeric Nanofibers}

\subsubsection{Natural Polymeric Nanofibers}

(1) Collagen Nanofibers. Collagen is a common element of the extracellular matrix (ECM) and plays an important structural role in the body, providing strength and support to the aorta, skin, bones, ligaments, and tendons, to name a few [31, 32]. Collagen is known to be the most promising biomaterials and has been found diverse applications in tissue engineering for its excellent biocompatibility and biodegradability. The crosslinking treatment to collagen has become one of the most important issues for the collagen-based scaffolds, due to the fast biodegrading rate and the low mechanical strength of the untreated collagen scaffold. By enzyme-mediated crosslinking mechanical support, helping to resist tissue strain is provided to adjacent collagen molecules [33]. Aside from naturally formed collagen fibers, collagen fibers can also be produced through the process of electrostatic spinning. Electrospun collagen, in particular, has great potential in scaffold engineering, because biomechanical structures formed from collagen have been shown to supply a substrate for cell adhesion, improving cell growth and differentiation [34], while the helicity and rigidity of the collagen molecule supply strength [35]. Scaffolds constructed from collagen blends have been studied in vitro for vascular graft applications and have shown potential as a treatment for skeletal muscle tissue defects. Some specific examples of implications of electrospun collagen will be presented in the following paragraph.

Bone is known to be mainly composed of collagen fibers and the oriented HA minerals. Teng et al. [36] fabricated collagen/hydroxyapatite composite nanofibers with an average 
diameter of about $60 \mathrm{~nm}$ successfully from the sol precursor via the electrospinning process. The electrospun fibrous scaffolds were compositionally and structurally similar to the natural system, which may be an ideal candidate for the bone regeneration engineering. Zhang et al. [37], fabricated bilayer microporous scaffold with collagen and electrospun poly-Llactic acid nanofibers (COL-nanofibers) successfully. Then they examined their adhesion, proliferation, and differentiation of mesenchymal stem cells which were cultured on the bi-layer scaffold. Moreover, they created osteochondral defects in rabbits and implanted with COL-nanofibers scaffold. They found that cells on COL-nanofibers scaffold exhibited more robust osteogenic differentiation what is more, implantation of COL-nanofibers scaffold seeded with cells induced more rapid subchondral bone emergence and better cartilage formation. They fully proved that the potential of using the bi-layer microporous COL-nanofibers scaffold for the treatment of deep osteochondral defects.

(2) Chitosan Nanofibers. Chitin, the second most abundant natural polysaccharide, is synthesized by a number of living organisms, widely found in the exoskeletons of arthropods, the shells of crustaceans, and the cuticles of insects. The polysaccharide consists of N-acetyl-Dglucosamine monomeric units, which are a major component of extracellular matrix macromolecules in the human body. Chitin occurs in nature as ordered microfibrils and is the major structural component in the exoskeleton of arthropods and cell walls of fungi and yeast. Chitin is not readily dissolved in common solvents; it is often converted to its more deacetylated derivative, chitosan (CS) [38, 39].

CS is often identified by its degree of deacetylation (DD), a percentage measurement of free amine groups along the chitosan backbone [40]. Because of its solubility in acidic, neutral, and alkaline solutions, chitosan is preferred over chitin for a wide range of applications. CS has recently attracted increasing interest both in research and development aspects, offering unique properties such as biologically renewability, biodegradability, biocompatibility, nonantigenicity, nontoxicity, and ease of solubility in organic acids. It is promoting cell adhesion, proliferation and differentiation and evokes a minimal foreign body reaction on implantation [41].

Due to these properties, CS is widely used for biomedical applications such as tissue engineering scaffolds, drug delivery, wound dressings, separation membranes and antibacterial coatings, stent coatings, and sensors [42]. In the recent years, electrospinning has been found to be a novel technique to produce chitosan nanofibers. It can produce polymer nanofibers with diameter in the range from several micrometers down to tens of nanometers, depending on the polymer and processing conditions. In the following paragraphs, we will give a brief introduction on bioactive applications of chitosan nanofibers and its composites nanofibers.

CS with silicate hybrids was synthesized with glycidoxypropyltrimethoxy silane (GPTMS), whose epoxy groups are considered to react with the amino groups of chitosan. The cross-linking density was around $80 \%$ regardless of the amount of silane [43]. The values of the mechanical parameters indicated that significant stiffening of the hybrids was obtained upon addition of the silane while full flexibility was retained. In addition, adhesion and proliferation of the MG63 osteoblasts cells cultured on the hybrid surface were improved compared to those on the pure chitosan membrane regardless of the silane concentration [43, 44]. Chitosan nanofibers was found to improve structural integrity of crosslink by silicate nanoparticles (laponite) films and to enhance the formation of a mineralized extracellular matrix and the differentiation of MC3T3-E1 preosteoblasts cells [45].

Swarnalatha et al. [46] indicated the attachment, viability and functionality of rat blood outgrowth endothelial cells and genetically modified r-blood outgrowth endothelial cells (rBOEC), which overexpress endothelial nitric oxide synthase, were investigated on poly(lactic acid) (PLA)-chitosan and PLA-chitosan-collagen nanofibrous scaffolds. Both cell types displayed good attachment and remained viable and functional on both scaffolds. Moreover, chitosan is an interesting biopolymer, because of its abundant production in nature, excellent biocompatibility, excellent antibacterial and anticorrosion properties, appropriate biodegradability, excellent physicochemical properties, and commercial availability at relatively low cost. It has been widely used as biomaterials in the pharmaceutical and medical fields as well as collagen, for example, tissue-engineering scaffolding. Chen et al. [47] ingeniously fabricated collagen-chitosan complex nanofibers by electrospinning, and the composites nanofibers are supposed to mimic the native extracellular matrix for tissue engineering and to develop functional nanobiomaterials. In a word, chitosan nanofibers may give the possibility of producing new materials for potential biomedical applications.

(3) Silk Fibroin Nanofibers. Many organisms produce silk. Silkworms and spiders are two main ones. Silkworm silk, is well known and has been of interest for at least 5000 years due to its importance as a textile fiber. Scaffolds for cellular growth made of silk fibroin (SF) demonstrate diverse applications in the field of tissue engineering [48]. Silk fibroin is extracted from the silk which is a natural polymer fibers, count for about $70 \%$ to $80 \%$ in the silk. SF is highly biocompatible and able to support appropriate cellular activity without eliciting rejection, inflammation, or immune activation in the host. SF structure is porous allowing the growing of cells, the exchange of nutrients, and growth factors and the production of ECM to enable communication between the cells.

Silk fibroin is extremely versatile and can be processed in very different formats, adequate for different tissue engineering needs. Silk fibroin cellular scaffolds can be constructed predominantly as hydrogels, $3 \mathrm{D}$ sponges, and mats of nanofibers obtained by electrospinning [49]. Electro spun mats of silk fibroin as well as other polymeric biomaterials are satisfactory for a high number of tissue engineering applications. Park et al. [50] successfully produced silk fibroin/chitin blend nanofibers. Li et al. [51] added bone morphogenetic protein-2 (BMP-2) into silk fibroin nanofibers mixing it into the spinning solution and successfully fabricated core-sheath nanofibers composed of PCL and silk fibroin using a singlenozzle technique and studied their potential applications in tissue engineering and drug release. Zarkoob et al. [52] 
discussed structure and morphology of electro spun silk and proved that electrospinning produced a nonwoven sheet of randomly arranged silk fibers with nanometer scale diameters. One of the promising applications of SF nanofibers in biomedical engineering is to be a scaffolding material for tissue engineering. It was reported that SF matrices can be used to culture fibroblasts and osteoblasts, as well as stem cells, and may enhance the adhesion, growth, and differentiation of the cells in a manner similar to that of collagen matrices.

Jeong et al. [53] prepared SF nanofibers by electrospinning and treated SF nanofibers with plasma in the presence of oxygen or methane gas to modify their surface characteristics. They examined the surface of nanofibers using contact angle measurements and XPS analysis. They demonstrated that the $\mathrm{O}_{2}$-treated $\mathrm{SF}$ nanofibers showed higher cellular activities for both normal human epidermal keratinocytes (NHEK) and fibroblasts (NHEF) than the untreated ones, and they think the difference in cellular activity might be due to the hydrophilicity of the SF nanofibers surface. Li et al. [51] generated core-sheath nanofibers composed of poly ( $\varepsilon$-caprolactone) (PCL) and SF blends via emulsion electrospinning. They characterized nanofibrous scaffolds by scanning electron microscopy (SEM), transmission electron microscopy (TEM), and other characterization methods. And they confirmed that the PCL/SF composite nanofibers greatly enhanced cells adhesion and proliferation. Furthermore, Jeong et al. proved the feasibility of PCL/SF nanofibers with core-sheath structure to be utilized as carrier for sustained drug release. Min et al. [54] fabricated SF nanofibers nonwovens for cell culture of normal human keratinocytes and fibroblasts using an electrospinning method. They also examined cell morphology on SF nanofibers using scanning electron microscopy, and the results indicated that the SF nanofibers may be a good candidate for the biomedical applications, such as wound dressing and scaffolds for tissue engineering. Obviously, we believe that SF nanofibrous scaffolds with desirable mechanical, biological, and especially bioactive properties and potential of controlled drugs release would be an ideal candidate for tissue engineering application.

2.2.2. Synthetic Polymeric Nanofibers. Synthetic polymeric nanofibers scaffolds generated by electrospinning have gained increasing popularity in the field of tissue engineering in the past few years $[55,56]$. Prepared by electrospinning method, they possess an extremely high porosity and surface area to volume ratio mimicking the features of the extracellular matrix that is critical for tissue regeneration [57]. Electrospinning is one of the approaches that allow the fabrication of synthetic materials into fibrous structures in the nanometer scale. Additionally, aligned nanofibers can be obtained by correct choice of the collector in the electrospinning equipment. Aligned nanofibers have been shown to direct cell migration, which plays a critical role in tissue regeneration [58]. Recently, synthetic polymeric nanofibers materials have been fabricated into nanometer scale structures in attempts to simulate the matrix environment in which cells can be accommodated to proliferate and differentiate towards desired lineages. We will exemplify the simulating effect on various cells of polymeric nanofibers.

Shao et al. [59] fabricated the random oriented and aligned electrically conductive nanofibers of biodegradable poly-DL-lactide (PLA) in which MWCNTs were embedded. In the course of culturing, electrical stimulation was added to explore their potential application in bone tissue engineering. They successfully proved aligned nanofibers as topographic cues could enhance the extension and direct the outgrowth of osteoblasts better than random fibers. In the presence of direct current (DC) of $100 \mathrm{~mA}$, the osteoblasts on all samples grew along the electrical current direction. Therefore, electrical stimulation with an appropriate DC value imparted on conductive substrate had great potential in application of bone tissue engineering. Similarly, Hsiao et al. [60] developed a mesh, consisting of aligned composite nanofibers of polyaniline (PANI) and poly(lactic-coglycolic acid) (PLGA). After doping, the electrospinning nanofibers could be transformed into a conductive form carrying positive charges, which could then attract negatively charged adhesive proteins (i.e., fibronectin and laminin) and enhance cell adhesion. Additionally, by examining a series of expression of cardiac-specific markers, such as cardiac troponin I (cTnI) and connexin 43 ( $\mathrm{Cx} 43)$, they proved that neonatal culture of rat cardiomyocytes on aligned composite nanofibers of polyaniline (PANI) and poly(lacticco-glycolic acid) (PLGA) promoted cell division and cell adhesion together via an electrical stimulation. All in all, electrically conductive polymeric nanofibers provide more possibility for treatment of myocardial infarction. Moreover, Xin et al. [61] seeded human MSCs on PLGA nanofibers sheets; then the majority of human mesenchymal stem cells (hMSCs) was viable and proliferating in PLGA nanofibers scaffolds up to the tested 14 days. Histological assays also revealed that hMSCs continuously differentiated into chondrogenic cells and osteogenic cells after 2-week incubation in PLGA nanofibers. Taken all into consideration, it represents continuous differentiation of hMSCs into chondrogenic and osteogenic cells in PLGA nanofibers scaffold. Additionally, synthetic polymeric nanofibers also have a great application in the nerve repair and regeneration. It remains a significant clinical problem that nerve injury cannot be directly repaired by end to end sutures. And a number of synthetic biopolymers, such as poly (L-lactic acid) (PLLA), and poly (lacticco-glycolic acid) (PLGA), poly(caprolactone) (PCL), have been utilized to construct nerve guidance conduit (NGCs) for nerve repair, but these degradable NGCs are still unsatisfactory as a result of their biological or mechanical properties. Nanofibrous scaffolds exhibit a great potential in curing nerve injury because of their high porosity, specific surface area and nano-scaled diameter. Wang et al. [62] fabricated aligned SF blended poly (L-lactic acid-co-e-caprolactone) (P (LLA$\mathrm{CL})$ ) nanofibrous scaffolds by electrospinning methods and then reeled into aligned nerve guidance conduits (NGC) to promote nerve regeneration successfully. 
2.3. Metallic Nanofibers. The increasing utilization of metallic materials for medical and dental devices is mainly due to their excellent mechanical properties. Among them, titanium and its alloys have been widely used as orthopedic and dental implant materials not only because of their compatible mechanical properties but also because of their good biocompatibility [63]. Due to the long duration of osseointegration, the stability of metal-based implants is still a problem. Nanostructured materials are prospective candidate to show an excellent biocompatibility due to their small dimensions and large surface to volume ratio [64]. Electrospinning was employed to fabricated metallic nanofibers. Moreover, the electrospun nanofibers are found to possess morphological resemblances such as high porosity and effective mechanical properties to the ECM of natural tissue. The implications of metallic nanofibers will be exemplified. For instance, Amna et al. [65] fabricated a kind of novel $\mathrm{Fe}_{3} \mathrm{O}_{4} / \mathrm{TiO}_{2}$ hybrid nanofibers using facile sol-gel electrospinning. They examined the morphological features of unexposed satellite cells and exposed to $\mathrm{Fe}_{3} \mathrm{O}_{4} / \mathrm{TiO}_{2}$ composite nanofibers with a phase contrast microscope, whereas the quantification of cell viability was carried out via confocal laser scanning microscopy, and the morphology of the cells attached to hybrid matrix was observed by Bio-SEM. They demonstrated that $\mathrm{Fe}_{3} \mathrm{O}_{4} / \mathrm{TiO}_{2}$ composite nanofibers scaffold with small diameters (approximately $200 \mathrm{~nm}$ ) can mimic the natural extracellular matrix well, support cell adhesion and growth, and also provide possibilities for diverse applications in the field of tissue engineering and regenerative medicine. Moreover, Lim et al. [66] investigated the nanofibrous modification of titanium implants and found that $\mathrm{TiO}_{2}$ nanofibers on titanium plates could be used for the surface modification of titanium implants to improve the osseo-integration. As it can be seen, metallic nanofibers have shown good biological performances and have a tremendously prospective in implant applications.

\section{Bioactivity of Nanotubes}

\subsection{Inorganic Nanotubes}

3.1.1. Carbon Nanotubes. CNTs were discovered in the late 1950s while the synthesis of CNTs was first reported in $1991[67,68]$. CNTs represent, along with fullerenes, the third allotropic crystalline form of carbon. There are several methods to produce CNT including arc discharge, laser ablation, and chemical vapor deposition [69]. There are two main types of nanotubes existing: the SWNT which are composed by a rolled monolayered graphene sheet and the MWNT which possess several graphitic concentric layers. The diameter varies from 0.4 to $2 \mathrm{~nm}$ for SWNT and from 1.4 to $100 \mathrm{~nm}$ for MWNT, while the length can reach several micrometers for both types [70].

The rediscovery of CNTs has opened new frontiers in the field of nanoscience and nanotechnology, as well as the material science [71-74]. They have received broad attention in the field of biomedicine; recent applications include biosensors, cancer therapy, drug delivery carrier, and regeneration medicine because of their unique electronic, physics properties, surface properties and their ability to traverse cellular membranes [75].

Because the nanostructured surfaces of CNTs show high (bio and cyto) compatibility, by promoting protein adsorption and enhancing subsequent cellular adhesion and tissue growth more than on traditional biomaterials' surfaces such as ceramics, titanium alloy, and biopolymers, many efforts have focused on the exploration of the bioactivity of both SWCNT and MWCNT in recent years.

CNTs have been used for hard-tissue application, such as the regeneration and repair of bones and teeth. It has been indicated that CNTs have the ability to induce osteogenic differentiation. Balani et al. [76] reinforced hydroxyapatite coatings by plasma-sprayed carbon nanotube and studied their interaction with human osteoblasts in vitro. Unrestricted growth of human osteoblast hFOB 1.19 cells has been observed near CNT regions claiming assistance by CNTs surfaces to promote cell growth and proliferation. Li et al. [5] have used MWCNTs to induce osteogenic differentiation of human adipose-derived MSCs in vitro and ectopic bone formation in vivo. In the study, the attachment, proliferation, osteogenic gene expression, ALP/DNA, protein/DNA, and mineralization of human adipose-derived stem cells cultured on MWNTs and graphite (GP) compacts with the same dimension were evaluated. The results show that MWNTs have the ability to induce the expression of ALP, cbfal, and COLIA1 genes while GP do not and that the MWNTs have the ability to induce ectopic bone formation in the dorsal musculature of ddY mice while GP do not. The results indicate that MWNTs may stimulate inducible cells to form inductive bone by concentrating more proteins, including bone-inducing proteins. According to the researches published, CNTs also have the ability to induce the maturation of osteoblast-like SaoS2 [77]. All in all, CNTs could induce cell adherence or growth by specific protein adsorption.

There are studies about soft-tissue application. Chen and Hsiue [78] demonstrated that carboxylated MWCNTs can induce and maintain neural differentiation of human bone marrow mesenchymal stem cells (hBMMSCs) without any exogenous differentiating factors, as evidenced by the protein expression. The data suggest that carboxylated MWCNTs play dual roles: promoting hBMMSC neural differentiation, including upregulating the neural growth factors and trapping these neural growth factors to create a suitable environment for long-term neural differentiation. Carboxylated MWCNT substrates may provide a method of posttransplantational spontaneous neural differentiation with low cytotoxicity for neuron injury repair.

Burke et al. [79] assessed the influence of functionalization of MWCNTs on thrombotic activity and compare the thrombotic activity of MWCNTs in vitro and in vivo. In vitro, MWCNTs activate the intrinsic pathway of coagulation as measured by activated partial thromboplastin time (aPTT) assays. Functionalization by amidation or carboxylation enhances this procoagulant activity. MWCNTs activate platelets in vitro, with amidated MWCNTs exhibiting greater platelet activation than carboxylated or pristine MWCNTs. However, contrasting trends are obtained in vivo, where 
functionalization tends to diminish rather than enhance procoagulant activity. In contrast, carboxylated MWCNTS exhibited little procoagulant tendency in vivo. The research gets the conclusion that the procoagulant tendencies of MWCNTs observed in vitro are not necessarily recapitulated in vivo. Further, functionalization can markedly attenuate the procoagulant activity of MWCNTs in vivo.

Most of the researches reported focus on the bioactivity of MWCNTs. Little notice is paid on the bioactivity of SWCNTs. In fact SWCNTs also have bioactivity. Bari et al. [80] studied the bioactivity of functionalized single walled carbon nanotubes (f-SWCNTs) in 2013. In their study, carboxylic acid functionalized single walled carbon nanotubes (f-SWCNT-COOH) were shown to support the viability and ex vivo expansion of freeze-thawed, nonenriched hematopoietic stem and progenitor cells (HSPC) in human umbilical cord blood-mononucleated cells (UCB$\mathrm{MNC}$ ). Their experiments in vitro showed that f-SWCNT$\mathrm{COOH}$ increased the viability of the $\mathrm{CD} 45+$ cells even without cytokine stimulation, while it reduced mitochondrial superoxides and caspase activity in CD45+ cells. In vivo data suggested that $\mathrm{f}-\mathrm{SWCNT}$-COOH expanded UCB-MNC could repopulate immunodeficient mice models with minimal acute or subacute symptoms of graft-versus-host disease (GVHD) and f-SWCNT-COOH dependent toxicity.

CNTs have certain toxicity, including the targeted to cells, organs, tissues, and the whole organism $[81,82]$. For instance, numerous in vitro and in vivo studies have shown that CNTs and/or associated contaminants or catalytic materials that arise during the production process may induce oxidative stress, prominent pulmonary inflammation, apoptosis in different cell types, and induction of cytotoxic effects on lungs [82]. While concerns that carbon nanotubes may be cytotoxic dampen the enthusiasm of this material for biomedical applications, new approaches are being developed to mitigate their toxicity. Through covalent modification and adding surfactants to the CNTs, the improvement of the living creature exploitation degree and reducing the biological toxicity can be achieved. Such strategies may prove to make carbon nanotubes safer and also more useful for tissue engineering. All in all, carbon nanotubes are promising materials because of their excellent mechanical and physical property and special bioactive property caused by high aspect ratio, and they can easily be tuned to reduce any cytotoxic effects.

3.1.2. Boron Nitride Nanotubes. Soon after the synthesis of carbon nanotubes in 1991, BNNTs were theoretically predicted and then fabricated successfully by arc-discharge method in 1995 [83]. In the following few years, a variety of methods, including laser ablation, ball-milling, substitution reaction, and chemical vapor deposition (CVD) were invented and adopted to synthesize BNNTs $[84,85]$.

A boron nitride nanotube can be imagined as a rolled up hexagonal $\mathrm{BN}$ layer or as a carbon nanotube in which alternating $\mathrm{B}$ and $\mathrm{N}$ atoms entirely substitute for $\mathrm{C}$ atoms. Similar to CNTs, BNNTs have chiralities, an important geometrical parameter, but, for them, the chiralities do not play an important role in determining electrical properties [86, 87]. The visible appearance is the most appealing difference between CNTs and BNNTs: the BNNTs are pure white, while the CNTs are black. The BNNTs' Young's modulus is a bit lower than that of CNTs, around 0.7-0.9 TPa [88].

No real applications of BNNTs were developed so far, let alone being developed in bio-application. However, some researches on BNNTs are closely related to applications, such as hydrogen storage, composite materials fabrication, biocompatibility tests. Most of the researches about BNNTs in bio-application focus on the exploitation of using BNNTs as smart and selective nanocarriers or composition used to reinforce matrix. These results of research related to bioactivity of BNNTs will be presented in the following sections.

Lahiri et al. [89] studied the cytocompatibility of BNNTs reinforced polylactide-polycaprolactone copolymer composite with osteoblasts and macrophages in vitro in 2010. The results show that the BNNT addition to PLC enhances the tensile strength. Interactions of the osteoblasts and macrophages with bare BNNTs prove them to be noncytotoxic. PLC-BNNT composites displayed increased osteoblast cell viability when compared to the PLC matrix. The addition of BNNTs also resulted in an increase in the expression levels of the Runx2 gene, the main regulator of osteoblast differentiation. And then Lahiri et al. [90] studied the biocompatibility of boron nitride nanotube reinforced hydroxyapatite composite to osteoblasts in 2011. HA-BNNT composite shows excellent mechanical property and wear resistance. Osteoblast proliferation and cell viability show no adverse effect of BNNT addition. In 2012, Ciofani et al. [91] studied the protein grafting, characterization, and interaction with human endothelial cells of the transferrin-conjugated boron nitride nanotubes. In their paper they reported on a covalent grafting of boron nitride nanotubes with human transferrin. After silanization of the nanotube wall, transferrin was linked to the nanotubes through carbamide binding. Their results demonstrated the possibility to enhance and target the cellular up-take of boron nitride nanotubes following appropriate functionalization approaches. In 2013, Del Turco et al. studied the cytocompatibility evaluation of glycol-chitosan coated boron nitridenanotubes in human endothelial cells [92]. They explored the effects of increasing concentrations of GC-BNNTs $(0-100 \mu \mathrm{g} / \mathrm{mL})$ on human vein endothelial cells (HUVECs), testing cell toxicity, proliferation, cytoskeleton integrity, cell activation, and DNA damage. The results showed that only a modest reduction in cell viability, tested by trypan blue assay, and the increased expression of vascular adhesion molecule-1, a marker of cell activation, were detected at the highest concentration used $(100 \mu \mathrm{g} / \mathrm{mL})$, while no significant changes were observed in cell viability, cytoskeleton integrity, or DNA damage.

3.1.3. Glass Tubes. Bioactive glasses hold significant promise for hard tissue engineering as they readily form a bioactive hydroxycarbonate apatite layer. Bioactive glasses can also be tailored to deliver ions at levels capable of promoting cell differentiation and osteogenesis $[93,94]$. Moreover, bioactive 
glasses have been shown to stimulate the secretion of angiogenic growth factors in vitro $[95,96]$.

The research about the bioactivity of glass tubes was done in 2012, by Xie et al. [97]. They studied the submicron bioactive glass tubes for bone tissue engineering using solgel and coaxial electrospinning techniques. Heavy mineral oil and gel solution were delivered by two independent syringe pumps during the coaxial electrospinning process. After removal of poly(vinyl pyrrolidone) and heavy mineral oil via calcination, submicron bioactive glass tubes were obtained. They examined the bioactivity of submicron bioactive glass tubes and fibers and evaluated their biocompatibility, using electrospun poly(e-caprolactone) fibers which were bioinactive materials for comparison. The bioactivity of the glass tubes was examined in a simulated body fluid, and they demonstrated the formation of hydroxyapatite-like minerals on both the outer and inner surfaces, while mineralization only occurred on their surface for bioactive glass solid fibers. In another words, the bioactive glass tubes had a faster induction of mineral formation than the solid fibers.

\subsection{Metallic Nanotubes}

3.2.1. $\mathrm{TiO}_{2}$ Nanotubes. Titanium (Ti) and its alloys have been broadly used as implantation materials due to their excellent properties such as high tensile strength, lower modulus, favorable biocompatibility, and better corrosion resistance compared to other metals alloys such as aluminum alloy, stainless steel, and Co-Cr alloys [98-103]. However, being bio-inert, they cannot bond with bone directly and do not actively stimulate initialization of bone formation on the surface at an early stage of implantation $[104,105]$. Recently, researchers have started to focus on surface modification of the implants in nano-scaled. Many works have been done to show that nanostructured surfaces can improve osteointegration of the implant [105-108].

Titanium dioxide $\left(\mathrm{TiO}_{2}\right)$ nanotubes have raised interest lately due to their high surface-to-volume ratio and the ability to provoke a greater degree of biological plasticity compared to conventional microstructures [109]. In addition, nanotubular $\mathrm{TiO}_{2}$ has been recognized as a promising biomaterial with proven thermal stability, corrosion resistance and biocompatibility. Methods to fabricate $\mathrm{TiO}_{2}$ nanotubes include the assisted-template method, electrochemical anodic oxidation [110], and hydrothermal treatment.

The role of surface nanotopography on cellular interaction and biological response of different types of cells is studied. The effects of $\mathrm{TiO}_{2}$ nanotubes on cellular response have been investigated using a variety of cell types, such as osteoblasts, fibroblasts, chondrocytes, endothelial cells, muscle cells, and epidermal keratinocytes.

Effect on Endothelial and Muscle Cells. Peng et al. [111] investigated the effects of nanotubular titanium oxide $\left(\mathrm{TiO}_{2}\right)$ surfaces on vascular cells. Endothelial cell (EC) and vascular smooth muscle cell (VSMC) response to nanotubes was investigated through immunofluorescence staining, scanning electron microscopy, 5-ethynyl-20-deoxyuridine proliferation assays, and prostaglandin I2 (PGI2) enzyme immunoassays. They found that the nanotubular surface significantly enhances EC proliferation and secretion of PGI2. The surface also results in a decrease in VSMC proliferation and increased expression of smooth muscle a-actin.

Effect on Chondrocyte. Brammer et al. [112] have altered $\mathrm{TiO}_{2}$ nanotube diameters from 30 to $100 \mathrm{~nm}$ by anodization and investigated the in vitro bovine cartilage chondrocyte (BCC) response to the different nanoscale dimensions. Initial SEM observations revealed that BCCs produced dense ECM fibrils on nanotubular substrates, which were lacking on flat Ti. Dense extracellular matrix fibrils were found on $\mathrm{TiO}_{2}$ nanotube substrate. $\mathrm{TiO}_{2}$ nanotube with diameter of $70 \mathrm{~nm}$ showed the highest glycosaminoglycan (GAG) secretion, aggrecan and collagen type II transcription level. This study demonstrates that $\mathrm{TiO}_{2}$ nanotube structures in the $70 \mathrm{~nm}$ diameter regime, already being an osseointegrating biomaterial, have significant and favorable effects on the extracellular matrix production of chondrocytes.

Effects on Osteoblasts. Zhao et al. [113] have systematically studied the effects on bone mesenchymal stem cell (MSC) osteogenic differentiation by various microscopic and biological characterization techniques. All the topographies are observed to induce MSC osteogenic differentiation in the absence of osteogenic supplements. The results show that the $\mathrm{TiO}_{2}$ nanotube surfaces significantly promote cell attachment and spread, collagen secretion, and ECM mineralization, as well as osteogenesis-related gene expression, the $\mathrm{TiO}_{2}$ nanotubes unambiguously demonstrate their excellent ability to support MSC proliferation and induce MSC osteogenic differentiation, especially those with the micropitted topography. Zhao et al. [114] then study the osteogenic activity of strontium loaded $\mathrm{TiO}_{2}$ nanotube arrays on titanium substrates in 2013. The results show that $\mathrm{Sr}$ incorporation enhances proliferation of rat MSCs on the NT structure. The titania nanotubes loaded with proper amount of incorporated Sr which shows excellent osteogenic properties is very attractive and has large clinical potential.

Enhancement of Blood Clotting. Roy et al. [115] held the idea that the main biological purpose of blood coagulation is formation of an obstacle to prevent blood loss of hydraulic strength sufficient to withstand the blood pressure. The ability to rapidly stem hemorrhage in trauma patients significantly impacts their chances of survival and hence is a subject of ongoing interest in the medical community. Herein, they report on the effect of biocompatible $\mathrm{TiO}_{2}$ nanotubes on the clotting kinetics of whole blood. $\mathrm{TiO}_{2}$ nanotubes $10 \mathrm{~mm}$ long were prepared by anodization of titanium in an electrolyte comprised of dimethyl sulfoxide and HF and then dispersed by sonication. Compared to pure blood, blood containing dispersed $\mathrm{TiO}_{2}$ nanotubes and blood in contact with gauze pads surface-decorated with nanotubes demonstrated significantly stronger clot formation at reduced clotting times. Similar experiments using nanocrystalline $\mathrm{TiO}_{2}$ nanoparticles showed comparatively weaker clot strengths and increased 
clotting times. The $\mathrm{TiO}_{2}$ nanotubes appear to act as a scaffold, facilitating fibrin formation. Our results suggest that application of a $\mathrm{TiO}_{2}$ nanotube functionalized bandage could be used to help stem or stop hemorrhage.

Effect on Other Cells. There are also researches about effect of CNTs on other cells including mesenchymal stem cell (MSC), fibroblast, and epidermal keratinocyte. $\mathrm{TiO}_{2}$ nanotubes with carbon-coated $\mathrm{TiO}_{2}$ nanotubes, probing osteogenic cell behavior, including osteoblast (bone cells) and mesenchymal stem cell (osteoprogenitor cells) interactions with the different surface chemistries $\left(\mathrm{TiO}_{2}\right.$ versus carbon). The roles played by the material surface chemistry of the nanotubes did not have an effect on the adhesion, growth, or morphology but had a major influence on the alkaline phosphatase (ALP) activity of osteoblast cells, with the original $\mathrm{TiO}_{2}$ chemistry having higher ALP levels. In addition, the different chemistries caused different levels of osteogenic differentiation in MSCs; however, it was the carbon-coated $\mathrm{TiO}_{2}$ nanotubes that had the greater advantage, with higher levels of osteodifferentiation [116].

The result about fibroblast and epidermal keratinocyte shows that nanotube topography is favorable for the growth and maintenance of dermal fibroblast but not for epidermal keratinocyte. Increased dermal fibroblast and decreased epidermal keratinocyte adhesion, proliferation, and differentiation were observed [116-118].

3.2.2. $\mathrm{ZrO}_{2}$ Nanotubes. During the last several decades, zirconium dioxide $\left(\mathrm{ZrO}_{2}\right)$ has become increasingly attractive to many researchers due to its excellent physical, mechanical, and chemical properties, such as chemical and thermal stability, corrosion resistance, and good biocompatibility [119]. It has been widely applied as sensors [120], catalyst supporters [121], and biomedical implants [122].

To this date, a large part of the interest has remained on titanium oxide $\left(\mathrm{TiO}_{2}\right)$ nanotubes because it is well known that titanium is a biocompatible orthopedic material which provides an excellent osseointegrative surface. In fact $\mathrm{ZrO}_{2}$ nanotubes have similar property to $\mathrm{TiO}_{2}$ nanotubes because of the similar self-assembled mechanism as $\mathrm{TiO}_{2}$ nanotubes, through an electrochemical anodization process $[123,124]$.

It is known that $\mathrm{TiO}_{2}$ nanotube layer could significantly enhance mesenchymal stem cell growth and differentiation. However, a study published in 2009 by Bauer and coworkers demonstrated that mesenchymal stem cells react in the same manner to $\mathrm{ZrO}_{2}$ nanotubes, AuPd-coated $\mathrm{TiO}_{2}$ nanotubes, and as-formed $\mathrm{TiO}_{2}$ nanotubes [125]. Subsequently, Frandsen et al. studied the cellular response to a unique vertically aligned, laterally spaced nanotube nanostructure made of $\mathrm{ZrO}_{2}$ fabricated by anodization in 2011 [126]. According to their study, the initial adhesion and spreading were considerably improved on the nanotube surface as compared to a flat zirconium $(\mathrm{Zr}$ ) surface without a nanostructure. The morphology of the adhered cells on the nanotube surface elicited a highly organized cytoskeleton with crisscross patterned actin, which was lacking on the flat Zr. Increased alkaline phosphatase activity levels and the formation of calcified extracellular matrix implied improved osteoblast functionality and mineralization on the nanotube substrate. Their study suggests that the $\mathrm{ZrO}_{2}$ nanotubes provided an enhanced osteoblast response and demonstrated their apparent role in providing a platform for bone growth. Besides, Wang and Luo $[119,123]$ used an effective way to prepare bioactive $\mathrm{ZrO}_{2}$ nanotubular arrays by a nodic oxidation in phosphate containing electrolyte. It is known that hydroxyapatite (HA) coating has been widely applied on metallic biomedical implants to enhance their biocompatibility. By this method, the surfaces of the nanotubular arrays so made contain a certain phosphate species, and these enhance the bioactivity for formation of HA coatings in simulated biological culture.

3.3. Bioactivity of Polymeric Nanotubes. There are also some other bioactive nanotubes except $\mathrm{CNTs}, \mathrm{TiO}_{2}$ nanotubes, and BNNTs, such as polymer nanotubes, ceramic nanotubes, and other nanotubes. For instance of polymer, which include natural polymer and synthesized polymer, when they are fabricated as nanotubes, some of them demonstrate excellent biocompatibility and bioactivity due to the high aspect ratio and surface area of nanostructure.

3.3.1. Chitin Nanotubes. Chitin is a promising polymer for biomedical applications because of its biocompatibility, biodegradability, and structural similarity to the glycosaminoglycans. Chitin and chitosan support nerve cell adhesion and neurite outgrowth. Freier et al. studied the chitin-based tubes for tissue engineering in the nervous system in 2007 [127]. Transparent chitin hydrogel tubes were synthesized from chitosan solutions using acylation chemistry and mold casting techniques, while the diameters of the tubes were in microscale. The results show that the chin-based tubes had the ability to support never cells adhesion and neurite outgrowth. If we fabricate chinbased tubes in nanoscale, the ability of supporting nerve cells adhesion and neurite outgrowth will be kept even enhanced for the high aspect ratio and surface area of nanotubes.

3.3.2. Chitosan Nanotubes. As it was mentioned above that CS is generally obtained by extensive deacetylation of chitin, a polysaccharide widely spread in nature. In the year 2007, Yang et al. studied the assembled alginate/chitosan nanotubes for biological application [128]. Biodegradable nanotubes were fabricated through the layer-by-layer (LbL) assembly technique of alternate adsorption of alginate (ALG) and chitosan onto the inner pores of polycarbonate template with the subsequent removal of the template. They fabricated the $(\mathrm{ALG} / \mathrm{CS})_{8}$ nanotubes with the dimension of about $400 \mathrm{~nm}$ and the wall thickness of $40 \mathrm{~nm}$ by alternate adsorption of negatively charged ALG and positively charged CHI onto the inner pores of $\mathrm{PC}$ template after removal of the template. They carried out the viability experiments of cells in the presence and absence of $(\mathrm{ALG} / \mathrm{CS})_{8}$ nanotubes. The results showed 
that the nanotubes had no influence upon the growth of cells and exhibit low cytotoxicity.

3.3.3. Poly(3,4-ethylene dioxythiophene) Nanotubes. Poly(3,4ethylene dioxythiophene) (PEDOT) is a $\pi$-conjugated polymer and polymerized oxidatively, either with appropriate oxidants (chemically) or with an electrochemical current, which has received a large number of interest for various applications. Different PEDOT morphologies coated on electrodes have been shown to have potential for improving the communication between bionic devices and living tissue $[129,130]$.

In 2013, Feng et al. studied the highly aligned poly(3,4ethylene dioxythiophene) (PEDOT) nano- and microscale tubes and fibers [130]. They reported a facile method to fabricate aligned poly(3,4-ethylene dioxythiophene) (PEDOT) tubes based on electrospinning and oxidative chemical polymerization. As it was reported that the aligned nanofiber assemblies fabricated by electrospinning could be used to direct neural cell growth in vitro [131], the PEDOT nanotubes may also have the same ability.

\section{The Possible Mechanism}

Among all the biomaterials mentioned above, some of the biomaterials are compositionally similar to the natural system such as collagen which is one of the components of nature bone, chitin, which consists of $\mathrm{N}$-acetyl-D-glucosamine monomeric units which are a major component of extracellular matrix macromolecules in the human body. By using these materials which are compositionally similar to the natural system, the biocompatibility and the bioactivity of biomedical materials can be improved. In fact, not all the materials used for biomedical application are compositionally similar to the natural system. The biocompatibility and bioactivity of biomedical materials can be also improved by the use of these materials which are not compositionally similar to the natural system but nanostructured such as CNTS, BNNTs, and $\mathrm{TiO}_{2}$ nanotubes. In another word, by the use of these nanofibers or nanotubes the biocompatibility and bioactivity of biomedical materials can be improved.

According to the researches mentioned above and studies we have done before $[5,71-74,77,82,106,108,132-$ 141], we present the possible mechanism of improving the biocompatibility and bioactivity of biomedical materials by using nano-scaled fibers or tubes as follows: the high aspect ratio and surface area of nanofibers and nanotubes enable nanomaterials to concentrate specific proteins or enzymes on the surface of materials to stimulate corresponding cell attachment, differentiation, or growth, performing desired function, generating appropriate beneficial cellular or tissue response in that specific situation, and improving the biocompatibility and bioactivity of biomedical materials. For instance, $\mathrm{TiO}_{2}$ nanotube surfaces promote cell attachment and spread, collagen secretion, and ECM mineralization, as well as osteogenesis-related gene expression $[109,111]$. The experiments that use MWCNTs to induce osteogenic differentiation of human adipose-derived MSCs in vitro and ectopic bone formation in vivo show that MWNTs may stimulate inducible cells to form inductive bone by concentrating more proteins, including bone-inducing proteins [76, 77]. When implanting the biocomposites which are reinforced by nanotubes and used as scaffolds for bone tissue engineering, more proteins concentrating on the surface of nanotubes can be found at first; then the cell adherence happens on the surface of nanotubes; the osteogenic differentiation happen following the cell adherence; finally, new tissue formed and new bone regenerated (Figure 1). It is the high aspect ratio and surface area of nano-scaled fibers and tubes that induce the specific protein absorption and cell adherence.

\section{Conclusions and Perspectives}

In this paper, nanofibers and nanotubes materials can improve the biocompatibility and bioactivity of biomedical materials, such as ceramic nanofibers/tubes, polymeric nanofibers/tubes, metallic nanofibers/tubes, and other nanomaterials. Most of nanomaterials show excellent biocompatibility, nontoxicity properties, especially for natural polymeric nanofibers. Carbon nanotubes may be cytotoxic, but it can be nontoxic by functionalized processing. Functionalized carbon nanofibers/tubes are shown to be more bioactive and biocompatible and applied to bone regeneration and neural repair successfully. Overall, carbon nanotubes are promising biomaterials because of their excellent mechanical and special bioactive property caused by high aspect ratio. Phosphate glass nanofibers/tubes are usually used in hard tissue applications, such as the regeneration and repair of bones and teeth using sol-gel and coaxial electrospinning techniques. As to glass fibers or tubes, hydroxyapatite-like minerals will be formed on the surfaces of hard-tissue after they are implanted in vivo, then improving bioactivity and biocompatibility of glass nanofibers/tubes. As with polymeric nanofibers/tubes, we classify it into natural and synthetic polymeric nanofibers/tubes. In natural polymeric nanofibers/tubes, we give a more detailed introduction about collagen, chitosan, and silk fibroin nanofibers/tubes. As natural polymers, they exhibit excellent biocompatibility, biodegradable properties, non-toxicity, and bioactivity. Due to these properties, these natural polymeric nanofibers/tubes are widely used for biomedical applications such as tissue engineering scaffolds, wound dressings, and many other biomedical aspects. Synthetic polymeric nanofibers/tubes are presented synthetically due to their various kinds. Importantly, we found synthetic polymeric nanofibers/tubes composites could direct the outgrowth of osteoblasts, promote myocardial cell differentiation, and stimulate nerve repair and regeneration. These bioactivity performances fully demonstrate synthetic polymeric nanofibers/tubes are excellently biocompatible and extremely promising biomaterials for the tissue engineering application. With respect to metallic nanofibers/tubes, we briefly introduce the bioactivity of $\mathrm{TiO}_{2}$ nanotubes, $\mathrm{Fe}_{3} \mathrm{O}_{4} / \mathrm{TiO}_{2}$ composite nanofibers. All in all, whether ceramic nanofibers/tubes, metallic nanofibers/tubes, 


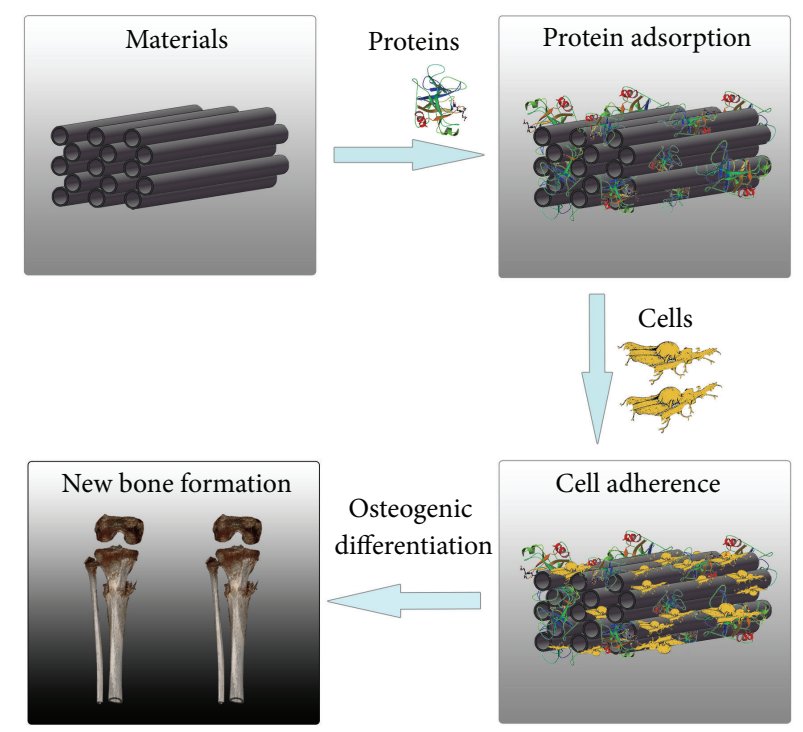

Figure 1: Schematic illustration of the possible mechanism by which nanomaterials may be superior to conventional materials when used for tissue regeneration. The high aspect ratio and surface area of nanomaterials enable them to concentrate specific proteins or enzymes to stimulate corresponding cell attachment, differentiation, or growth, performing desired functions, generating appropriate beneficial cellular or tissue response in the specific situations, improving the biocompatibility and bioactivity of the materials. The high aspect ratio and surface area of some nanotubes efficiently induce specific protein adsorption, which promotes cellular functions and thereby achieve new bone formation.

or polymeric nanofibers/tubes, nanomaterials can all promote cells (such as osteoblasts, fibroblasts, endothelial cells, and muscle cells) division and proliferation under specified conditions.

Additionally, we infer that unique microstructure of nanofibers and nanotubes enables nanomaterials to concentrate specific proteins or enzymes on the surface of materials to stimulate corresponding cell attachment, differentiation, or growth, performing its desired function with respect to a medical therapy, without eliciting undesirable local or systemic effects in the recipient or beneficiary of that therapy, but generating the most appropriate beneficial cellular or tissue response in that specific situation and optimising the clinically relevant performance of that therapy [1]. The biocompatibility of biomedical materials can be improved following the use of bioactive materials with unique microstructure of nanofibers and nanotubes. In another word, by using nanostructured fibers or tubes, the biocompatibility and bioactivity can be improved. So nanofibers and nanotubes materials are excellent candidates for tissue repair. That the nano-scaled fibers or tubes may concentrate specific proteins on the surface of materials to stimulate corresponding cell differentiation or growth is inferred to be the mechanism of improving the biocompatibility and bioactivity of biomedical materials.

In the future, a further study on the mechanism of improving the biocompatibility and bioactivity by using nano-scale fibers or tubes should be carried on, as well as the specific proteins absorption on the surface of biomedical materials. More methods of inducting nonbioactive materials to be bioactive could be developed. The functionalized biomaterials that could improve the bioactivity and biocompatibility will be applied for biomedicine application in quantity.

\section{Conflict of Interests}

The authors declare that they have no financial or personal relationship with any people or organization that may inappropriately influence their work; there is no professional or commercial interest of any kind in all the commercial identities mentioned in our paper.

\section{Acknowledgments}

The authors acknowledge the financial supports from the National Basic Research Program of China (973 Program, no. 2011CB710901), the National Natural Science Foundation of China (no. 31000431, 31370959, 81101348, and 10925208), the Beijing Nova Program (no. 2010B011), Program for New Century Excellent Talents (NCET) in University from Ministry of Education of China, State Key Laboratory of New Ceramic and Fine Processing (Tsinghua University), Natural Science Foundation of Guangdong Province, China (no. 10451051501004727 and 10151051501000107), Guangdong Medical Scientific Research project (no. A2013385), Doctoral Fund of Ministry of Education, China (20114433120004), outstanding person fund of Zhujiang Hospital, International Joint Research Center of Aerospace Biotechnology and Medical Engineering, Ministry of Science and Technology of China, and the 111 Project (no. B13003). The authors 
would like to announce that Professor Bo Yu (E-mail: gzyubo@gmail.com) is another corresponding author of this paper.

\section{References}

[1] D. F. Williams, "On the mechanisms of biocompatibility," Biomaterials, vol. 29, no. 20, pp. 2941-2953, 2008.

[2] D. F. Williams, "Definitions in biomaterials: progress in biomedical engineering," Biomaterials, vol. 10, pp. 216-238, 1987.

[3] T. Kokubo and H. Takadama, "How useful is SBF in predicting in vivo bone bioactivity?" Biomaterials, vol. 27, no. 15, pp. 29072915, 2006.

[4] H.-W. Kim, H.-E. Kim, and J. C. Knowles, "Production and potential of bioactive glass nanofibers as a next-generation biomaterial," Advanced Functional Materials, vol. 16, no. 12, pp. 1529-1535, 2006.

[5] X. Li, H. Liu, X. Niu et al., "The use of carbon nanotubes to induce osteogenic differentiation of human adipose-derived MSCs in vitro and ectopic bone formation in vivo," Biomaterials, vol. 33, no. 19, pp. 4818-4827, 2012.

[6] H. Chen and S. Dong, "Direct electrochemistry and electrocatalysis of horseradish peroxidase immobilized in sol-gelderived ceramic-carbon nanotube nanocomposite film," Biosensors and Bioelectronics, vol. 22, no. 8, pp. 1811-1815, 2007.

[7] C. Xiang, Y. Zou, L.-X. Sun, and F. Xu, "Direct electron transfer of cytochrome $\mathrm{c}$ and its biosensor based on gold nanoparticles/room temperature ionic liquid/carbon nanotubes composite film," Electrochemistry Communications, vol. 10, no. 1, pp. 3841, 2008.

[8] M. E. Ghica, R. Pauliukaite, O. Fatibello-Filho, and C. M. A. Brett, "Application of functionalised carbon nanotubes immobilised into chitosan films in amperometric enzyme biosensors," Sensors and Actuators B, vol. 142, no. 1, pp. 308-315, 2009.

[9] Y. Wang, X. Wang, B. Wu et al., "Dispersion of single-walled carbon nanotubes in poly(diallyldimethylammonium chloride) for preparation of a glucose biosensor," Sensors and Actuators B, vol. 130, no. 2, pp. 809-815, 2008.

[10] K. Lafdi, W. Fox, M. Matzek, and E. Yildiz, "Effect of carbon nanofiber heat treatment on physical properties of polymeric nanocomposites: part I," Journal of Nanomaterials, vol. 2007, Article ID 52729, 6 pages, 2007.

[11] S. Niyogi, M. A. Hamon, H. Hu et al., "Chemistry of singlewalled carbon nanotubes," Accounts of Chemical Research, vol. 35, no. 12, pp. 1105-1113, 2002.

[12] N. M. Rodriguez, A. Chambers, and R. T. K. Baker, "Catalytic engineering of carbon nanostructures," Langmuir, vol. 11, no. 10, pp. 3862-3866, 1995.

[13] V. Vamvakaki, K. Tsagaraki, and N. Chaniotakis, "Carbon nanofiber-based glucose biosensor," Analytical Chemistry, vol. 78, no. 15, pp. 5538-5542, 2006.

[14] L. Wu, J. Lei, X. Zhang, and H. Ju, "Biofunctional nanocomposite of carbon nanofiber with water-soluble porphyrin for highly sensitive ethanol biosensing," Biosensors and Bioelectronics, vol. 24, no. 4, pp. 644-649, 2008.

[15] A. Greiner and J. H. Wendorff, "Electrospinning: a fascinating method for the preparation of ultrathin fibers," Angewandte Chemie, vol. 46, no. 30, pp. 5670-5703, 2007.

[16] X. Tang, Y. Liu, H. Hou, and T. You, "Electrochemical determination of L-Tryptophan, L-Tyrosine and L-Cysteine using electrospun carbon nanofibers modified electrode," Talanta, vol. 80, no. 5, pp. 2182-2186, 2010.

[17] D. Liang, B. S. Hsiao, and B. Chu, "Functional electrospun nanofibrous scaffolds for biomedical applications," Advanced Drug Delivery Reviews, vol. 59, no. 14, pp. 1392-1412, 2007.

[18] C. Huang, B. Lucas, C. Vervaet et al., "Unbreakable codes in electrospun fibers: digitally encoded polymers to stop medicine counterfeiting," Advanced Materials, vol. 22, no. 24, pp. 26572662, 2010.

[19] N. M. Rodriguez, "Review of catalytically grown carbon nanofibers," Journal of Materials Research, vol. 8, no. 12, pp. 3233-3250, 1993.

[20] R. L. Price, M. C. Waid, K. M. Haberstroh, and T. J. Webster, "Selective bone cell adhesion on formulations containing carbon nanofibers," Biomaterials, vol. 24, no. 11, pp. 1877-1887, 2003.

[21] I. Rajzer, R. Kwiatkowski, W. Piekarczyk, W. Binias, and J. Janicki, "Carbon nanofibers produced from modified electro spun PAN/hydroxyapatite precursors as scaffolds for bone tissue engineering," Materials Science and Engineering C, vol. 32, no. 8, pp. 2562-2569, 2012.

[22] A. Carranza-Bencano, J. R. Armas-Padrón, M. Gili-Miner, and M. A. Lozano, "Carbon fiber implants in osteochondral defects of the rabbit patella," Biomaterials, vol. 21, no. 21, pp. 2171-2176, 2000.

[23] K. L. Elias, R. L. Price, and T. J. Webster, "Enhanced functions of osteoblasts on nanometer diameter carbon fibers," Biomaterials, vol. 23, no. 15, pp. 3279-3287, 2002.

[24] L. Yang, L. Zhang, and T. J. Webster, "Nanobiomaterials: state of the art and future trends," Advanced Engineering Materials, vol. 13, no. 6, pp. B197-B217, 2011.

[25] J. L. McKenzie, M. C. Waid, R. Shi, and T. J. Webster, "Decreased functions of astrocytes on carbon nanofiber materials," Biomaterials, vol. 25, no. 7-8, pp. 1309-1317, 2004.

[26] J. M. Gomez-Vega, A. Hozumi, H. Sugimura, and O. Takai, "Ordered mesoporous silica coatings that induce apatite formation in vitro," Advanced Materials, vol. 13, pp. 822-825, 2001.

[27] V. Maquet, A. R. Boccaccini, L. Pravata, I. Notingher, and R. Jérôme, "Porous poly $(\alpha$-hydroxyacid)/Bioglass composite scaffolds for bone tissue engineering. I: preparation and in vitro characterisation," Biomaterials, vol. 25, no. 18, pp. 4185-4194, 2004.

[28] S. Madhugiri, A. Dalton, J. Gutierrez, J. P. Ferraris, and K. J. Balkus Jr., "Electrospun MEH-PPV/SBA-15 composite nanofibers using a dual syringe method," Journal of the American Chemical Society, vol. 125, no. 47, pp. 14531-14538, 2003.

[29] W. Shi, W. Lu, and L. Jiang, "The fabrication of photosensitive self-assembly Au nanoparticles embedded in silica nanofibers by electrospinning," Journal of Colloid and Interface Science, vol. 340, no. 2, pp. 291-297, 2009.

[30] M. Kouhia, M. Morshed, J. Varshosaz, and M. Hossein Fathi, "Poly ( $\varepsilon$-caprolactone) incorporated bioactive glass nanoparticles and simvastatin nanocomposite nanofibers: preparation, characterization and in vitro drug release for bone regeneration applications," Chemical Engineering Journal, vol. 228, pp. 10571065, 2013.

[31] F. H. Silver, J. W. Freeman, and D. Devore, "Viscoelastic properties of human skin and processed dermis," Skin Research and Technology, vol. 7, no. 1, pp. 18-23, 2001.

[32] M. G. Dunn and F. H. Silver, "Viscoelastic behavior of human connective tissues: relative contribution of viscous and elastic 
components," Connective Tissue Research, vol. 12, no. 1, pp. 5970, 1983.

[33] D. R. Eyre, M. A. Paz, and P. M. Gallop, "Cross-linking in collagen and elastin," Annual Review of Biochemistry, vol. 53, pp. 717-748, 1984.

[34] H. K. Kleinman, R. J. Klebe, and G. R. Martin, "Role of collagenous matrices in the adhesion and growth of cells," Journal of Cell Biology, vol. 88, no. 3, pp. 473-485, 1981.

[35] H. Lodish, A. Berk, L. Zipursky, P. Matsudaira, D. Baltimore, and J. Darnell, Molecular Cell Biology, W.H. Freeman, New York, NY, USA, 2000.

[36] S.-H. Teng, E.-J. Lee, P. Wang, and H.-E. Kim, "Collagen/hydroxyapatite composite nanofibers by electrospinning," Materials Letters, vol. 62, no. 17-18, pp. 3055-3058, 2008.

[37] S. Zhang, L. Chen, Y. Jiang et al., "Bi-layer collagen/microporous electrospun nanofiber scaffold improves the osteochondral regeneration," Acta Biomaterialia, vol. 9, pp. 7236-7247, 2013.

[38] M. Rinaudo, "Chitin and chitosan: properties and applications," Progress in Polymer Science, vol. 31, no. 7, pp. 603-632, 2006.

[39] C. K. S. Pillai, W. Paul, and C. P. Sharma, "Chitin and chitosan polymers: chemistry, solubility and fiber formation," Progress in Polymer Science, vol. 34, no. 7, pp. 641-678, 2009.

[40] K. Kurita, "Chemistry and application of chitin and chitosan," Polymer Degradation and Stability, vol. 59, no. 1-3, pp. 117-120, 1998.

[41] R. A. A. Muzzarelli, "Chitins and chitosans for the repair of wounded skin, nerve, cartilage and bone," Carbohydrate Polymers, vol. 76, no. 2, pp. 167-182, 2009.

[42] M. N. V. Ravi Kumar, "A review of chitin and chitosan applications," Reactive and Functional Polymers, vol. 46, no. 1, pp. 1-27, 2000.

[43] R. A. A. Muzzarelli, "Chitosan composites with inorganics, morphogenetic proteins and stem cells, for bone regeneration," Carbohydrate Polymers, vol. 83, no. 4, pp. 1433-1445, 2011.

[44] Y. Shirosaki, K. Tsuru, S. Hayakawa et al., "Physical, chemical and in vitro biological profile of chitosan hybrid membrane as a function of organosiloxane concentration," Acta Biomaterialia, vol. 5, no. 1, pp. 346-355, 2009.

[45] A. K. Gaharwar, P. J. Schexnailder, Q. Jin, C.-J. Wu, and G. Schmidt, "Addition of chitosan to silicate cross-linked PEO for tuning osteoblast cell adhesion and mineralization," ACS Applied Materials and Interfaces, vol. 2, no. 11, pp. 3119-3127, 2010.

[46] B. Swarnalatha, S. L. Nairb, K. T. Shalumon et al., "Poly(lactic acid)-chitosan-collagen composite nanofibers assubstrates for blood outgrowth endothelial cells," International Journal of Biological Macromolecules, vol. 58, pp. 220-224, 2013.

[47] Z. Chen, X. Mo, and F. Qing, "Electrospinning of collagenchitosan complex," Materials Letters, vol. 61, no. 16, pp. 34903494, 2007.

[48] Y. Wang, H.-J. Kim, G. Vunjak-Novakovic, and D. L. Kaplan, "Stem cell-based tissue engineering with silk biomaterials," Biomaterials, vol. 27, no. 36, pp. 6064-6082, 2006.

[49] X. Zhang, M. R. Reagan, and D. L. Kaplan, "Electrospun silk biomaterial scaffolds for regenerative medicine," Advanced Drug Delivery Reviews, vol. 61, no. 12, pp. 988-1006, 2009.

[50] K. E. Park, S. Y. Jung, S. J. Lee, B.-M. Min, and W. H. Park, "Biomimetic nanofibrous scaffolds: preparation and characterization of chitin/silk fibroin blend nanofibers," International Journal of Biological Macromolecules, vol. 38, no. 3-5, pp. 165173, 2006.
[51] L. Li, H. Li, Y. Qian et al., "Electrospun poly ( $\varepsilon$ caprolactone)/silk fibroin core-sheath nanofibers and their potential applications in tissue engineering and drug release," International Journal of Biological Macromolecules, vol. 49, no. 2, pp. 223-232, 2011.

[52] S. Zarkoob, R. K. Eby, D. H. Reneker, S. D. Hudson, D. Ertley, and W. W. Adams, "Structure and morphology of electrospun silk nanofibers," Polymer, vol. 45, no. 11, pp. 3973-3977, 2004.

[53] L. Jeong, I.-S. Yeo, H. N. Kim et al., "Plasma-treated silk fibroin nanofibers for skin regeneration," International Journal of Biological Macromolecules, vol. 44, no. 3, pp. 222-228, 2009.

[54] B.-M. Min, G. Lee, S. H. Kim, Y. S. Nam, T. S. Lee, and W. H. Park, "Electrospinning of silk fibroin nanofibers and its effect on the adhesion and spreading of normal human keratinocytes and fibroblasts in vitro," Biomaterials, vol. 25, no. 7-8, pp. 12891297, 2004.

[55] J. Xie, X. Li, and Y. Xia, "Putting electrospun nanofibers to work for biomedical research," Macromolecular Rapid Communications, vol. 29, no. 22, pp. 1775-1792, 2008.

[56] J. D. Schiffman and C. L. Schauer, "A review: electrospinning of biopolymer nanofibers and their applications," Polymer Reviews, vol. 48, no. 2, pp. 317-352, 2008.

[57] A. S. Badami, M. R. Kreke, M. S. Thompson, J. S. Riffle, and A. S. Goldstein, "Effect of fiber diameter on spreading, proliferation, and differentiation of osteoblastic cells on electrospun poly(lactic acid) substrates," Biomaterials, vol. 27, no. 4, pp. 596606, 2006.

[58] J. Venugopal, S. Low, A. T. Choon, and S. Ramakrishna, "Interaction of cells and nanofiber scaffolds in tissue engineering," Journal of Biomedical Materials Research B, vol. 84, no. 1, pp. 34-48, 2008.

[59] S. Shao, S. Zhou, L. Li et al., "Osteoblast function on electrically conductive electrospun PLA/MWCNTs nanofibers," Biomaterials, vol. 32, no. 11, pp. 2821-2833, 2011.

[60] C. W. Hsiao, M. Y. Bai, Y. Chang et al., "Electrical coupling of isolated cardiomyocyte clusters grown on aligned conductive nanofibrous meshes for their synchronized beating," Biomaterials, vol. 34, pp. 1063-1072, 2013.

[61] X. Xin, M. Hussain, and J. J. Mao, "Continuing differentiation of human mesenchymal stem cells and induced chondrogenic and osteogenic lineages in electrospun PLGA nanofiber scaffold," Biomaterials, vol. 28, no. 2, pp. 316-325, 2007.

[62] C.-Y. Wang, K.-H. Zhang, C.-Y. Fan, X.-M. Mo, H.-J. Ruan, and F.-F. Li, "Aligned natural-synthetic polyblend nanofibers for peripheral nerve regeneration," Acta Biomaterialia, vol. 7, no. 2, pp. 634-643, 2011.

[63] H. J. Rack and J. I. Qazi, "Titanium alloys for biomedical applications," Materials Science and Engineering C, vol. 26, no. 8, pp. 1269-1277, 2006.

[64] A. M. Ektessabi, T. Otsuka, Y. Tsuboi et al., "Preliminary experimental results on mapping of the elemental distribution of the organic tissues surrounding titanium-alloy implants," Nuclear Instruments and Methods in Physics Research B, vol. 109110, pp. 278-283, 1996.

[65] T. Amna, M. Shamshi Hassan, H. van Ba, M. S. Khil, H. K. Lee, and I. H. Hwang, "Electro spun $\mathrm{Fe}_{2} \mathrm{O}_{4} / \mathrm{TiO}_{2}$ hybrid nanofibers and their in vitro biocompatibility: prospective matrix for satellite cell adhesion and cultivation," Materials Science and Engineering C, vol. 33, no. 2, pp. 707-713, 2013.

[66] J. Lim, B. Yu, K. M. Woo, and Y. K. Lee, "Immobilization of $\mathrm{TiO}_{2}$ nanofibers on titanium plates for implant applications," Applied Surface Science, vol. 255, no. 5, pp. 2456-2460, 2008. 
[67] R. Bacon, "Growth, structure, and properties of graphite whiskers," Journal of Applied Physics, vol. 31, no. 2, pp. 283-290, 1960.

[68] S. Iijima, "Helical microtubules of graphitic carbon," Nature, vol. 354, no. 6348, pp. 56-58, 1991.

[69] C. E. Baddour and C. Briens, "Carbon nanotube synthesis: a review," International Journal of Chemical Reactor Engineering, vol. 3, 2005.

[70] C. Klumpp, K. Kostarelos, M. Prato, and A. Bianco, "Functionalized carbon nanotubes as emerging nanovectors for the delivery of therapeutics," Biochimica et Biophysica Acta, vol. 1758, no. 3, pp. 404-412, 2006.

[71] X. Li, Y. Yang, Y. Fan, Q. Feng, F. Z. Cui, and F. Watari, "Biocomposites reinforced by fibers or tubes as scaffolds for tissue engineering or regenerative medicine," Journal of Biomedical Materials Research A, 2013.

[72] X. M. Li, L. Wang, Y. B. Fan et al., "Nanostructured scaffolds for bone tissue engineering," Journal of Biomedical Materials Research A, vol. 101, pp. 2424-2435, 2013.

[73] X. Li, H. Gao, M. Uo et al., "Effect of carbon nanotubes on cellular functions in vitro," Journal of Biomedical Materials Research A, vol. 91, no. 1, pp. 132-139, 2009.

[74] X. Li, X. Liu, J. Huang, Y. Fan, and F.-Z. Cui, "Biomedical investigation of CNT based coatings," Surface and Coatings Technology, vol. 206, no. 4, pp. 759-766, 2011.

[75] N. Saito, Y. Usui, K. Aoki et al., "Carbon nanotubes: biomaterial applications," Chemical Society Reviews, vol. 38, no. 7, pp. 18971903, 2009.

[76] K. Balani, R. Anderson, T. Laha et al., "Plasma-sprayed carbon nanotube reinforced hydroxyapatite coatings and their interaction with human osteoblasts in vitro," Biomaterials, vol. 28, no. 4, pp. 618-624, 2007.

[77] X. Li, H. Gao, M. Uo et al., "Maturation of osteoblast-like SaoS induced by carbon nanotubes," Biomedical Materials, vol. 4, no. 1, Article ID 015005, 2009.

[78] Y.-S. Chen and G.-H. Hsiue, "Directing neural differentiation of mesenchymal stem cells by carboxylated multiwalled carbon nanotubes," Biomaterials, vol. 34, pp. 4936-4944, 2013.

[79] A. R. Burke, R. N. Singh, D. L. Carroll et al., "Determinants of the thrombogenic potential of multiwalled carbon nanotubes," Biomaterials, vol. 32, no. 26, pp. 5970-5978, 2011.

[80] S. Bari, P. P. Chu, A. Lim et al., "Protective role of functionalized single walled carbon nanotubes enhance ex vivo expansion of hematopoietic stem and progenitor cells in human umbilical cord blood," Nanomedicine, 2013.

[81] X. Zhao and R. Liu, "Recent progress and perspectives on the toxicity of carbon nanotubes at organism, organ, cell, and biomacromolecule levels," Environment International, vol. 40, no. 1, pp. 244-255, 2012.

[82] X. M. Li, L. Wang, Y. B. Fan, Q. Fang, and F. Z. Cui, "Biocompatibility and toxicity of nanoparticles and nanotubes," Journal of Nanomaterials, vol. 2012, Article ID 548389, 19 pages, 2012.

[83] N. G. Chopra, R. J. Luyken, K. Cherrey et al., "Boron nitride nanotubes," Science, vol. 269, no. 5226, pp. 966-967, 1995.

[84] L. Lia, X. Liu, L. Li, and Y. Chen, "High yield BNNTs synthesis by promotion effect of milling-assisted precursor," Microelectronic Engineering, vol. 110, pp. 256-259, 2013.

[85] B. Zhong, L. Song, X. X. Huang, G. W. Wen, and L. Xia, "Synthesis of boron nitride nanotubes with $\mathrm{SiC}$ nanowire as template," Materials Research Bulletin, vol. 46, no. 9, pp. 15211523, 2011.
[86] B. Akdim, X. Duan, W. W. Adams, and R. Pachter, "A comparative theoretical study of carbon and boron-nitride single-wall nanotubes," in Proceedings of the Nanotechnology Conference and Trade Show (Nanotech '03), pp. 147-148, February 2003.

[87] B. Akdim, X. Duan, W. W. Adams, and R. Pachter, "A comparative theoretical study of carbon and boron-nitride single-wall nanotubes," in Proceedings of the 3rd International Conference on Computational Nanoscience and Technology, 2003.

[88] C. Zhi, Y. Bando, C. Tang, and D. Golberg, "Boron nitride nanotubes," Materials Science and Engineering R, vol. 70, no. 3-6, pp. 92-111, 2010.

[89] D. Lahiri, F. Rouzaud, T. Richard et al., "Boron nitride nanotube reinforced polylactide-polycaprolactone copolymer composite: mechanical properties and cytocompatibility with osteoblasts and macrophages in vitro," Acta Biomaterialia, vol. 6, no. 9, pp. 3524-3533, 2010.

[90] D. Lahiri, V. Singh, A. P. Benaduce, S. Seal, L. Kos, and A. Agarwal, "Boron nitride nanotube reinforced hydroxyapatite composite: mechanical and tribological performance and invitro biocompatibility to osteoblasts," Journal of the Mechanical Behavior of Biomedical Materials, vol. 4, no. 1, pp. 44-56, 2011.

[91] G. Ciofani, S. Del Turco, G. G. Genchia et al., "Transferrinconjugated boron nitride nanotubes: protein grafting, characterization, and interaction with human endothelial cells," International Journal of Pharmaceutics, vol. 436, pp. 444-453, 2012.

[92] S. Del Turco, G. Ciofani, V. Cappello et al., "Cytocompatibility evaluation of glycol-chitosan coated boron nitridenanotubes in human endothelial cells," Colloids and Surfaces B, vol. 111, pp. 142-149, 2013.

[93] D. Xynos, A. J. Edgar, L. D. K. Buttery et al., "Gene-expression profiling of human osteoblasts following treatment with the ionic products of Bioglass 45S5 dissolution," Journal of Biomedical Materials Research, vol. 55, pp. 151-157, 2001.

[94] G. Jell and M. M. Stevens, "Gene activation by bioactive glasses," Journal of Materials Science, vol. 17, no. 11, pp. 997-1002, 2006.

[95] O. Leppäranta, M. Vaahtio, T. Peltola et al., "Antibacterial effect of bioactive glasses on clinically important anaerobic bacteria in vitro," Journal of Materials Science, vol. 19, no. 2, pp. 547-551, 2008.

[96] R. M. Day, "Bioactive glass stimulates the secretion of angiogenic growth factors and angiogenesis in vitro," Tissue Engineering, vol. 11, no. 5-6, pp. 768-777, 2005.

[97] J. Xie, E. R. Blough, and C.-H. Wang, "Submicron bioactive glass tubes for bone tissue engineering," Acta Biomaterialia, vol. 8, no. 2, pp. 811-819, 2012.

[98] W. Q. Yu, J. Qui, L. Xu, and F. Q. Zhang, "Corrosion behaviors of $\mathrm{TiO}_{2}$ nanotube layers on titanium in Hank's solution," Biomedical Materials, vol. 4, no. 6, Article ID 065012, 2009.

[99] G. J. Chen, Z. Wang, H. Bai, J. M. Li, and H. Cai, "A preliminary study on investigating the attachment of soft tissue onto microarc oxidized titanium alloy implants," Biomedical Materials, vol. 4, no. 1, Article ID 015017, 2009.

[100] W. Q. Yu, Y. L. Zhang, X. Q. Jiang, and F. Q. Zhang, "In vitro behavior of MC3T3-E1 preosteoblast with different annealing temperature titania nanotubes," Oral Diseases, vol. 16, no. 7, pp. 624-630, 2010.

[101] K. Das, S. Bose, and A. Bandyopadhyay, " $\mathrm{TiO}_{2}$ nanotubes on Ti: influence of nanoscale morphology on bone cell-materials interaction," Journal of Biomedical Materials Research A, vol. 90, no. 1, pp. 225-237, 2009. 
[102] H. Kim, S.-H. Choi, J.-J. Ryu, S.-Y. Koh, J.-H. Park, and I.-S. Lee, "The biocompatibility of SLA-treated titanium implants," Biomedical Materials, vol. 3, no. 2, Article ID 25011, 2008.

[103] Z. X. Chen, Y. Takao, W. X. Wang, T. Matsubara, and L. M. Ren, "Surface characteristics and in vitro biocompatibility of titanium anodized in a phosphoric acid solution at different voltages," Biomedical Materials, vol. 4, no. 6, Article ID 065003 , 2009.

[104] X. Hu, H. Shen, K. Shuai et al., "Surface bioactivity modification of titanium by $\mathrm{CO}_{2}$ plasma treatment and induction of hydroxyapatite: in vitro and in vivo studies," Applied Surface Science, vol. 257, no. 6, pp. 1813-1823, 2011.

[105] T. J. Webster, C. Ergun, R. H. Doremus, R. W. Siegel, and R. Bizios, "Enhanced functions of osteoblasts on nanophase ceramics," Biomaterials, vol. 21, no. 17, pp. 1803-1810, 2000.

[106] X. Li, C. A. van Blitterswijk, Q. Feng, F. Cui, and F. Watari, “The effect of calcium phosphate microstructure on bone-related cells in vitro," Biomaterials, vol. 29, no. 23, pp. 3306-3316, 2008.

[107] T. J. Webster and J. U. Ejiofor, "Increased osteoblast adhesion on nanophase metals: $\mathrm{Ti}_{1} \mathrm{Ti}_{6} \mathrm{Al}_{4} \mathrm{~V}$, and CoCrMo," Biomaterials, vol. 25, no. 19, pp. 4731-4739, 2004.

[108] X. Li, H. Liu, X. Niu et al., "Osteogenic differentiation of human adipose-derived stem cells induced by osteoinductive calcium phosphate ceramics," Journal of Biomedical Materials Research $B$, vol. 97, no. 1, pp. 10-19, 2011.

[109] S. Oh, K. S. Brammer, Y. S. J. Li et al., "Stem cell fate dictated solely by altered nanotube dimension," Proceedings of the National Academy of Sciences of the United States of America, vol. 106, no. 7, pp. 2130-2135, 2009.

[110] K. Vasilev, Z. Poh, K. Kant, J. Chan, A. Michelmore, and D. Losic, "Tailoring the surface functionalities of titania nanotube arrays," Biomaterials, vol. 31, no. 3, pp. 532-540, 2010.

[111] L. Peng, M. L. Eltgroth, T. J. LaTempa, C. A. Grimes, and T. A. Desai, "The effect of $\mathrm{TiO}_{2}$ nanotubes on endothelial function and smooth muscle proliferation," Biomaterials, vol. 30, no. 7, pp. 1268-1272, 2009.

[112] K. S. Brammer, S. Oh, C. J. Frandsen, S. Varghese, and S. Jin, "Nanotube surface triggers increased chondrocyte extracellular matrix production," Materials Science and Engineering C, vol. 30, no. 4, pp. 518-525, 2010.

[113] L. Zhao, L. Liu, Z. Wu, Y. Zhang, and P. K. Chu, "Effects of micropitted/nanotubular titania topographies on bone mesenchymal stem cell osteogenic differentiation," Biomaterials, vol. 33, no. 9, pp. 2629-2641, 2012.

[114] L. Zhao, H. Wang, K. Huo et al., "The osteogenic activity of strontium loaded titania nanotube arrays on titanium substrates," Biomaterials, vol. 34, pp. 19-29, 2013.

[115] S. C. Roy, M. Paulose, and C. A. Grimes, "The effect of $\mathrm{TiO}_{2}$ nanotubes in the enhancement of blood clotting for the control of hemorrhage," Biomaterials, vol. 28, pp. 4667-4672, 2007.

[116] A. W. Tan, B. Pingguan-Murphy, R. Ahmad, and S. A. Akbar, "Review of titania nanotubes: fabrication and cellular response," Ceramics International, vol. 38, pp. 4421-4435, 2012.

[117] T. Sjöström, G. Lalev, J. P. Mansell, and B. Su, "Initial attachment and spreading of MG63 cells on nanopatterned titanium surfaces via through-mask anodization," Applied Surface Science, vol. 257, no. 10, pp. 4552-4558, 2011.

[118] J. M. Macak, H. Tsuchiya, L. Taveira, S. Aldabergerova, and P. Schmuki, "Smooth anodic $\mathrm{TiO}_{2}$ nanotubes," Angewandte Chemie, vol. 44, no. 45, pp. 7463-7465, 2005.
[119] L.-N. Wang and J.-L. Luo, "Fabrication and formation of bioactive anodic zirconium oxide nanotubes containing presynthesized hydroxyapatite via alternative immersion method," Materials Science and Engineering C, vol. 31, no. 4, pp. 748-754, 2011.

[120] R. Zhang, X. Zhang, and S. Hu, "Nanocrystalline $\mathrm{ZrO}_{2}$ thin films as electrode materials using in high temperature-pressure chemical sensors," Materials Letters, vol. 60, no. 25-26, pp. 31703174, 2006.

[121] S. Xu and X. Wang, "Highly active and coking resistant $\mathrm{Ni} / \mathrm{CeO}_{2}-\mathrm{ZrO}_{2}$ catalyst for partial oxidation of methane," Fuel, vol. 84, no. 5, pp. 563-567, 2005.

[122] X. Li, Y. Fan, and F. Watari, "Current investigations into carbon nanotubes for biomedical application," Biomedical Materials, vol. 5, no. 2, Article ID 022001, 2010.

[123] L.-N. Wang and J.-L. Luo, "Enhancing the bioactivity of zirconium with the coating of anodized $\mathrm{ZrO}_{2}$ nanotubular arrays prepared in phosphate containing electrolyte," Electrochemistry Communications, vol. 12, no. 11, pp. 1559-1562, 2010.

[124] S. Berger, J. Faltenbacher, S. Bauer, and P. Schmuki, "Enhanced self-ordering of anodic $\mathrm{ZrO}_{2}$ nanotubes in inorganic and organic electrolytes using two-step anodization," Physica Status Solidi, vol. 2, no. 3, pp. 102-104, 2008.

[125] S. Bauer, J. Park, J. Faltenbacher, S. Berger, K. von der Mark, and P. Schmuki, "Size selective behavior of mesenchymal stem cells on $\mathrm{ZrO}_{2}$ and $\mathrm{TiO}_{2}$ nanotube arrays," Integrative Biology, vol. 1, no. 8-9, pp. 525-532, 2009.

[126] C. J. Frandsen, K. S. Brammer, K. Noh et al., "Zirconium oxide nanotube surface prompts increased osteoblast functionality and mineralization," Materials Science and Engineering C, vol. 31, no. 8, pp. 1716-1722, 2011.

[127] T. Freier, R. Montenegro, H. S. Koh, and M. S. Shoichet, "Chitinbased tubes for tissue engineering in the nervous system," Biomaterials, vol. 26, no. 22, pp. 4624-4632, 2005.

[128] Y. Yang, Q. He, L. Duan, Y. Cui, and J. Li, "Assembled alginate/chitosan nanotubes for biological application," Biomaterials, vol. 28, no. 20, pp. 3083-3090, 2007.

[129] J. Yang, K. Lipkin, and D. C. Martin, "Electrochemical fabrication of conducting polymer poly(3,4-ethylenedioxythiophene) (PEDOT) nanofibrils on microfabricated neural prosthetic devices," Journal of Biomaterials Science, vol. 18, no. 8, pp. 10751089, 2007.

[130] Z.-Q. Feng, J. Wu, W. Cho et al., "Highly aligned poly (3, 4-ethylene dioxythiophene)(PEDOT) nano- and microscale fibers and tubes," Polymer, vol. 54, pp. 702-708, 2013.

[131] V. J. Mukhatyar, M. Salmerón-Sánchez, S. Rudra et al., "Role of fibronectin in topographical guidance of neurite extension on electrospun fibers," Biomaterials, vol. 32, no. 16, pp. 3958-3968, 2011.

[132] X. M. Li, X. H. Liu, M. Uo, Q. L. Feng, F. Z. Cui, and F. Watari, "Investigation on the mechanism of the osteoinduction for calcium phosphate," Bone, vol. 43, pp. S111-S112, 2008.

[133] X. M. Li and Q. L. Feng, "Dynamic rheological behaviors of the bone scaffold reinforced by chitin fibres," Materials Science Forum, vol. 475-479, pp. 2387-2390, 2005.

[134] X. Li, X. Liu, W. Dong et al., "In vitro evaluation of porous poly(L-lactic acid) scaffold reinforced by chitin fibers," Journal of Biomedical Materials Research B, vol. 90, no. 2, pp. 503-509, 2009.

[135] X. M. Li, Y. Huang, L. S. Zheng et al., "Effect of substrate stiffness on the functions of rat bone marrow and adipose tissue 
derived mesenchymal stem cells in vitro," Journal of Biomedical Materials Research A, 2013.

[136] X. Li, Q. Feng, W. Wang, and F. Cui, "Chemical characteristics and cytocompatibility of collagen-based scaffold reinforced by chitin fibers for bone tissue engineering," Journal of Biomedical Materials Research B, vol. 77, no. 2, pp. 219-226, 2006.

[137] X. Li, Q. Feng, and F. Cui, "In vitro degradation of porous nanohydroxyapatite/collagen/PLLA scaffold reinforced by chitin fibres," Materials Science and Engineering C, vol. 26, no. 4, pp. 716-720, 2006.

[138] X. Li and Q. Feng, "Porous poly-L-lactic acid scaffold reinforced by chitin fibers," Polymer Bulletin, vol. 54, no. 1-2, pp. 47-55, 2005.

[139] X. M. Li, X. H. Liu, G. P. Zhang et al., "Repairing $25 \mathrm{~mm}$ bone defect using fibres reinforced scaffolds as well as autograft bone," Bone, vol. 43, pp. S94-S94, 2008.

[140] X. Li, Q. Feng, Y. Jiao, and F. Cui, "Collagen-based scaffolds reinforced by chitosan fibres for bone tissue engineering," Polymer International, vol. 54, no. 7, pp. 1034-1040, 2005.

[141] X. Li, Q. Feng, X. Liu, W. Dong, and F. Cui, "Collagen-based implants reinforced by chitin fibres in a goat shank bone defect model," Biomaterials, vol. 27, no. 9, pp. 1917-1923, 2006. 

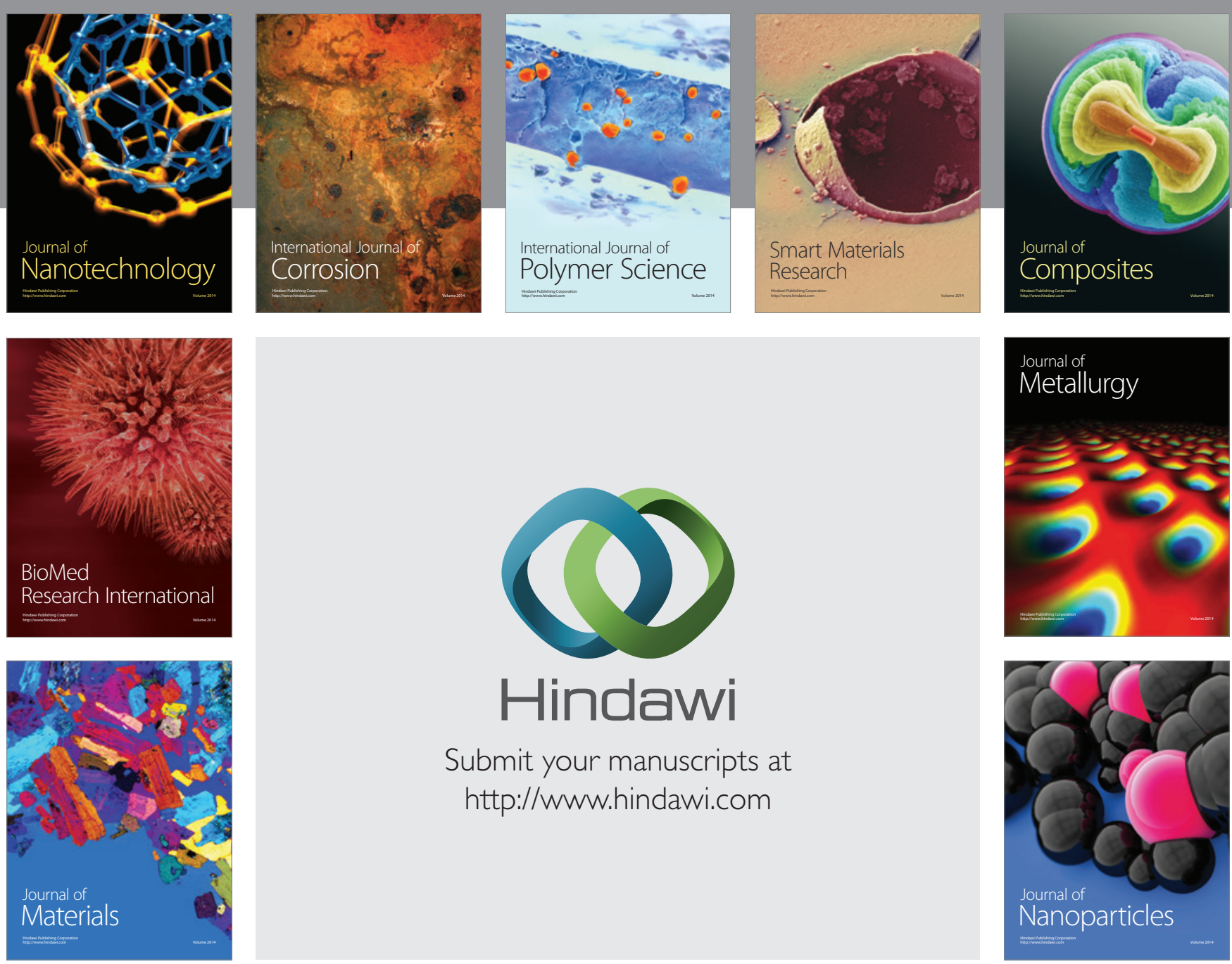

Submit your manuscripts at http://www.hindawi.com
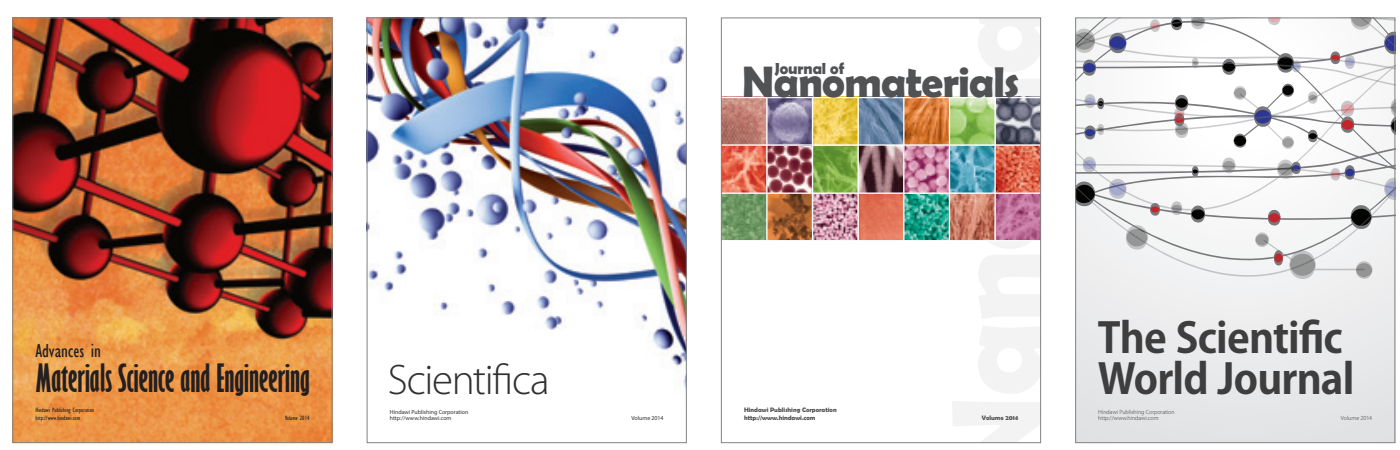

\section{The Scientific World Journal}
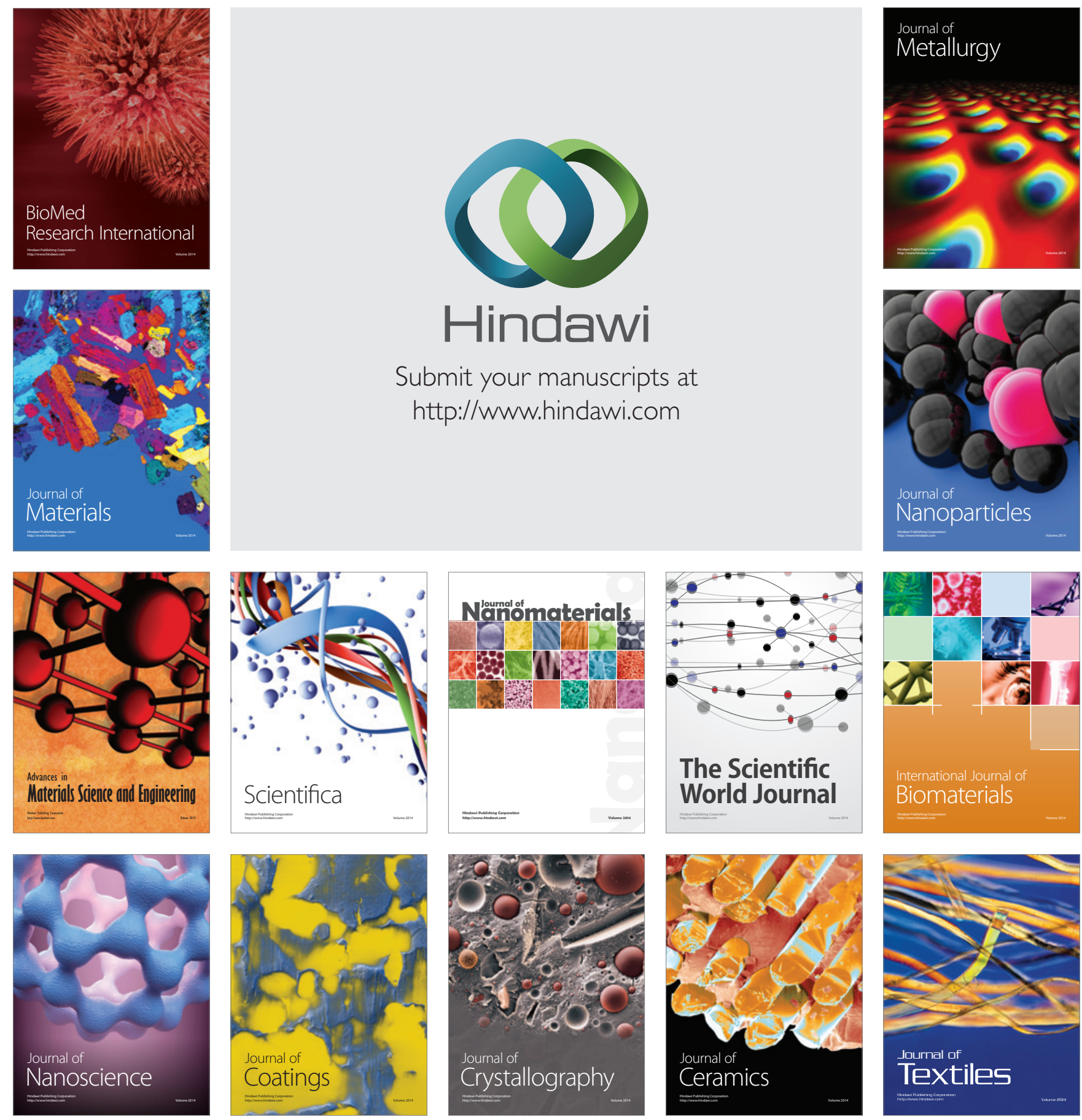